\title{
La eficiencia técnica de las universidades públicas del Perú
}

\author{
Marco Antonio Tello Miranda \\ JosÉ OVIDIO FLORES GUTIÉRREZ 2
}

\begin{abstract}
RESUMEN
Las deficiencias en la calidad de la educación superior figuran entre las principales debilidades del Perú según el ranking mundial de competitividad. Por ello, en este estudio se determinó la eficiencia técnica (ET) de las universidades públicas peruanas mediante el análisis envolvente de datos (DEA) y, además, se analizaron las relaciones entre la experiencia y calidad de las instituciones, y la ET. El estudio abarcó las 42 universidades públicas registradas para el año 2016, a la cuales se les midieron un insumo y dos productos. Se concluyó que las universidades públicas peruanas presentan funciones de producción de rendimientos constantes a escala (ETG promedio $=56.8 \%$ ), lo que sirvió de base para clasificar a las instituciones en cuatro categorías según sus orientaciones estratégicas (alta o baja) hacia la investigación o masificación educativa; asimismo, se comprobó que la ET no se relaciona con la experiencia o la calidad de la institución educativa.
\end{abstract}

Palabras clave: universidad; análisis envolvente de datos (DEA); benchmarking; calidad; experiencia.

\section{INTRODUCCIÓN}

Algunas corrientes teóricas en el ámbito económico aseveran que el porvenir de la economía de un país se fundamenta en la inversión y el desarrollo de la educación, la ciencia y la tecnología (Abdurakhmanova et al., 2020; Macilwain, 2010), lo cual implica que la educación es una variable relevante para el desarrollo de cualquier nación, toda vez que el capital humano resulta crucial para el crecimiento socio-económico y, por ende, para el desarrollo sostenible (Wodon, 2019; Blecich, 2020). Por ello, la política educativa de un país debe tener entre sus prioridades incrementar la calidad educativa en todos sus eslabones, coordinando políticas públicas para optimizar la infraestructura, así como la calidad de la capacitación y desempeño docente, entre otras importantes metas (Buckle y Creedy, 2019; Zhao, 2020).

La medición y la mejora de la eficiencia en el desenvolvimiento de las universidades se considera un tema relevante en los países desarrollados (Kumar y Thakur, 2019; Jiang et al., 2020) y de especial interés en la gerencia pública (Ayaviri y Zamora, 2016). De manera similar, las universidades públicas en los países latinoamericanos reconocen la necesidad de cambios y adaptación a las nuevas tendencias de la educación superior a nivel mundial, que imponen crecientes exigencias en busca de mayor eficiencia y la calidad en los servicios ofrecidos (Torres et al., 2019).

Según el ranking mundial de competitividad 2019, Perú ocupó el puesto 65 y se mantienen las principales debilidades en indicadores de los pilares: instituciones, universidades no licenciadas, infraestructura, educación, y capacidad de innovación, entre otros (Schwab, 2019). Asimismo, diversos autores han reportado deficiencias en la educación superior peruana (Lavalle y de Nicolas, 2017; Nunez y Cornejo, 2018), por ello, la presente

Bachiller en Ingeniería Química por la Universidad Nacional Mayor de San Marcos (Lima, Perú). Actualmente, es docente en el CERSEU de la Facultad de Ingeniería Industrial de la UNMSM. (Lima, Perú).

ORCID: https://orcid.org/0000-0002-4759-5772

Autor de correspondencia: marco.tello@unmsm.edu.pe

2 Doctor en Ingeniería por la Universidad de Valladolid (España). Actualmente, es profesor de posgrado en la Universidad Nacional Mayor de San Marcos. (Lima, Perú).

ORCID: https://orcid.org/0000-0001-5019-2635

E-mail: floresjoseovidio@gmail.com 
investigación busca determinar la eficiencia técnica (ET) en universidades públicas peruanas mediante la técnica matemática conocida como análisis envolvente de datos (DEA, por sus siglas en inglés), utilizada para medir y evaluar los resultados obtenidos en unidades productivas por medio de la comparación de sus niveles de productividad (Sigler, 2004; Rojas, 2010; Torres et al. , 2018). Adicionalmente, se determinan las relaciones existentes entre la experiencia y la calidad de las instituciones, y la eficiencia técnica.

La educación superior es de vital importancia en el desarrollo sostenible de los países, razón por la cual la eficiencia de su sistema debe ser considerada un tema prioritario. En este contexto, la presente investigación pretende determinar la eficiencia técnica (ET) de universidades públicas del Perú y, también, analizar las relaciones entre la experiencia y calidad de las instituciones, y la ET.

Entre las contribuciones más importantes de este trabajo se presenta un modelo DEA de un solo insumo y dos productos que representan las variables relevantes para analizar el sistema, lo cual es particularmente útil en contextos de información escasa o limitada-muy común en países en vías de desarrollo. Con los resultados del modelo DEA se elabora una herramienta sencilla y de fácil ejecución denominada MOEIM (Matriz de orientación estratégica a la investigación y masificación educativa), la cual proporciona una visión global del posicionamiento de las universidades en cuanto a su eficiencia relativa. Esto es relevante para orientar la toma de decisiones tanto a nivel de las universidades como de las instituciones que formulan y ejecutan políticas públicas enfocadas en la educación superior. Esta herramienta se complementa con el plan benchmarking basado en la técnica DEA.

\section{Análisis Envolvente de Datos (DEA)}

Según Buitrago et al. (2017), el DEA es una técnica que sirve para medir la eficiencia relativa en organizaciones educativas; además, es importante destacar que se utilizó por primera vez para evaluar colegios norteamericanos (Rhodes, 1978). Entre sus fortalezas destaca su precisión para medir la "unidad de toma de decisiones" sometida a evaluación, así como sus entradas y salidas; de manera que, una vez aplicada la técnica, se pueden clasificar entre unidades eficientes e ineficientes, y asignar pares de referencia con el fin de proporcionar guías para mejorar las unidades ineficientes y establecer metas para la correcta utilización de recursos.
Con base en un estudio sobre las universidades públicas de España, Salas-Velasco (2020) indica que el DEA puede ser un buen instrumento para la evaluación comparativa de la educación superior. En este caso, el DEA puede determinar, mediante el empleo de insumos y productos, instituciones técnicamente eficientes que funcionarían como referentes para clasificar universidades y, además, permitiría identificar los factores que inciden en la eficiencia técnica de las universidades evaluadas.

Castañeda (2019) indica que el DEA representa una técnica de programación matemática muy difundida creada por Charnes et al. (1978) y mejorada por Banker et al. (1984) para evaluar retornos variables de escala. EI DEA generaliza la medida de eficiencia técnica de Farrell (1957) para evaluar la eficiencia relativa de unidades pares con referente a múltiples medidas de rendimiento (Charnes et al., 1994; Cooper et al., 2006). Las organizaciones o unidades que son evaluadas con el DEA se denominan "unidades de toma de decisiones" (DMU en inglés) y según Cooper et al. (2006) una DMU se considera eficiente cuando ninguna otra DMU puede producir más salidas usando una cantidad igual o menor de entradas.

La ET se orienta a la maximización de los resultados de la DMU con base en los recursos utilizados (Campoverde et al., 2019). De esta manera se tiene que una universidad es eficiente cuando logra obtener niveles máximos de productos (o salidas) para un determinado nivel de dotación de insumos o, por el contrario, es capaz de minimizar el nivel de insumos aplicados para alcanzar un determinado nivel de producción. El principal aporte de la técnica DEA consiste en establecer de manera empírica o práctica un patrón referencial mediante una frontera, con la cual se compara la DMU, para saber si es o no eficiente, por ello se establece una eficiencia que es relativa, donde la estimación de la frontera constituye una de las vías para estimar la ET. De manera que Farrell (1957) aportó un método para calcular los índices, y separar técnicamente la eficiencia en técnica y asignativa, formulando preceptos sobre los rendimientos constantes a escala de la tecnología y una isocuanta convexa en el origen cuya pendiente es positiva.

En resumen, para realizar un análisis de eficiencia se define la "función de producción", la cual señala la máxima cantidad de producto (salida) generado por cada insumo (entrada), y se destacan las características tanto del proceso como del sector al cual pertenecen las DMU analizadas. 
Esta caracterización debe abarcar la tecnología aplicada en el proceso productivo, y las entradas y salidas del sistema, para asociar la función de producción con las variables que reflejan la tecnología y posibilitan la producción más eficiente en las DMU. Este enfoque puede entenderse en relación a las salidas (outputs) o entradas (inputs), de manera que una aplicación orientada a los outputs (como en la presente investigación) mostraría la máxima producción que se puede lograr con una combinación particular de factores; mientras que un análisis orientado a los inputs revelaría los requerimientos mínimos de insumos, combinados en una determinada proporción, para alcanzar un nivel determinado de productos (Escalona, 2013).

La eficiencia técnica se puede también denominar eficiencia técnica global (ETG) y está compuesta por la eficiencia técnica pura (ETP) y eficiencia de escala (EE), de manera que ETG = ETP x EE. Por tanto, para determinar si la tecnología de producción aplicada por las universidades presenta rendimientos variables a escala se aplican los dos modelos: rendimientos constantes a escala (RCE), que corresponde a la frontera ETG; y rendimientos variables a escala (RVE), que corresponde a la frontera ETP (Blecich, 2020). En el caso se detecten diferencias entre las dos mediciones para una universidad en particular, se asume que existen ineficiencias de escala, cuyos valores se atribuyen a la discrepancia entre la medición del RCE y del RVE. Así, la medición de la eficiencia de escala se obtiene como un resultado del cociente entre la medida de eficiencia técnica global y la medida de la eficiencia técnica pura. En resumen, la ETP evalúa la eficiencia técnica de la universidad como resultado específico de la gestión de la organización sin considerar el tamaño de la misma (Martin y Roman, 2010), mientras que las ineficiencias de escala son pérdidas ocasionadas por el hecho de no funcionar con el tamaño de producción óptimo. Entonces, se infiere que una universidad puede ser técnicamente eficiente $y$, aun así, tiene la posibilidad de mejorar su desempeño si decide explotar economías de escala (Coelli et al., 1998, p. 4).

De manera que las universidades eficientes serán aquellas que logren valores de $100 \%$ (o 1) para un determinado tipo de rendimiento (RCE o RVE) con el cual se esté comparando y, por ello, se considera que están localizadas sobre la frontera de producción o forman parte de ella. En contraste, valores por debajo del $100 \%(100 \%>x \geq 0)$ indican ineficiencias y, por ello, se ubicarán por debajo de la frontera de producción.

\section{Sistema de educación superior del Perú}

Arias (2019) indicó que a fines del 2019 la Superintendencia Nacional de Educación Superior Universitaria (SUNEDU) debió haber concluido el proceso de licenciamiento de las universidades peruanas que faltaban. La investigación es uno de los indicadores de calidad y es un requisito para el licenciamiento de universidades. Esta se debe hacer profesionalmente en estas casas de estudio y, dado que es escasa, es la principal dificultad que presentan algunas universidades.

En el Perú, la educación universitaria no se considera obligatoria y, por tanto, representa una opción educativa para las personas que completan el sistema de educación básica y se proponen proseguir estudios de tipo profesional, artísticos o técnicos (Díaz, 2008). De manera que la demanda del servicio de educación universitaria puede ser estimada en función de la cantidad de personas que, una vez completada la secundaria, concretan su aspiración de continuar estudios superiores postulando a cualquier institución educativa o, de manera más restrictiva, en función de los aspirantes que postulan e ingresan.

\section{METODOLOGÍA}

El enfoque de la investigación es cuantitativo, de tipo aplicada, tiene un alcance descriptivo y correlacional, con diseño no experimental y transversal. La muestra incluyó las 42 universidades públicas peruanas registradas para el año 2016 (Tabla 1), cuyos datos fueron obtenidos de la página web de cada universidad y también proporcionados por la SUNEDU (2018).

Se empleó como único insumo el presupuesto en millones (MM) de soles de cada universidad y dos productos: número de investigadores adscritos al programa Renacyt (antiguo Regina) y número de estudiantes de pregrado, maestría y doctorado matriculados, con base en un modelo propuesto por Ramírez y Alfaro (2013), el cual fue ligeramente modificado para medir la función de investigación. Otros insumos, como por ejemplo número de docentes, tienen alta correlación en el presupuesto en las universidades públicas. Asimismo, en esta investigación no se pudo especificar la cantidad de artículos en revistas indexadas por universidad, tal como se mencionó anteriormente, debido a que no están disponibles.

Las variables a correlacionar con la ET fueron la antigüedad (años), que refleja la experiencia de la 
Tabla 1. Listado de las 42 universidades públicas peruanas objeto de estudio.

\begin{tabular}{|c|c|c|}
\hline Universidad & Siglas & $\begin{array}{c}\text { Cuadrante } \\
\text { (matriz estratégica)* }\end{array}$ \\
\hline Universidad Nacional Mayor de San Marcos & UNMSM & 2 \\
\hline Universidad Nacional Agraria La Molina & UNALM & 2 \\
\hline Universidad Nacional de Ingeniería & UNI & 2 \\
\hline Universidad Nacional de San Antonio Abad del Cusco & UNSAAC & 3 \\
\hline Universidad Nacional de Trujillo & UNT & 2 \\
\hline Universidad Nacional de San Agustín & UNSA & 1 \\
\hline Universidad Nacional Santiago Antúnez de Mayolo & UNASAM & 4 \\
\hline Universidad Nacional del Altiplano & UNA & 1 \\
\hline Universidad Nacional de la Amazonía Peruana & UNAP & 2 \\
\hline Universidad Nacional Pedro Ruíz Gallo & UNPRG & 4 \\
\hline Universidad Nacional Federico Villarreal & UNFV & 4 \\
\hline Universidad Nacional del Callao & UNAC & 4 \\
\hline Universidad Nacional Hermilio Valdizán & UNHEVAL & 4 \\
\hline Universidad Nacional Jorge Basadre Grohmann & UNJBG & 3 \\
\hline Universidad Nacional de Cajamarca & UNC & 3 \\
\hline Universidad Nacional de Piura & UNP & 4 \\
\hline Universidad Nacional Autónoma de Chota & UNACH & 3 \\
\hline Universidad Nacional de San Martín & UNSM & 3 \\
\hline Universidad Nacional de San Cristóbal de Huamanga & UNSCH & 4 \\
\hline Universidad Nacional Agraria de la Selva & UNAS & 2 \\
\hline Universidad Nacional Daniel Alcídes Carrión & UNDAC & 4 \\
\hline Universidad Nacional de Tumbes & UNTumbes & 2 \\
\hline Universidad Nacional del Centro del Perú & UNCP & 4 \\
\hline Universidad Nacional San Luis Gonzaga & UNICA & 3 \\
\hline Universidad Nacional de Huancavelica & UNH & 3 \\
\hline Universidad Nacional de Educación Enrique Guzmán y Valle & UNE & 3 \\
\hline Universidad Nacional José Faustino Sánchez Carrión & UNFJFSC & 4 \\
\hline Universidad Nacional Micaela Bastidas de Apurímac & UNAMBA & 1 \\
\hline Universidad Nacional Toribio Rodríguez de Mendoza de Amazonas & UNTRM & 2 \\
\hline Universidad Nacional del Santa & UNS & 2 \\
\hline Universidad Nacional de Ucayali & UNU & 3 \\
\hline Universidad Nacional José María Arguedas & UNAJMA & 3 \\
\hline Universidad Nacional Amazónica de Madre de Dios & UNAMAD & 4 \\
\hline Universidad Nacional de Moquegua & UNAM & 3 \\
\hline Universidad Nacional Intercultural de la Amazonía & UNIA & 3 \\
\hline Universidad Nacional de Cañete & UNDC & 3 \\
\hline Universidad Nacional de Jaén & UNJ & 4 \\
\hline Universidad Nacional Tecnológica de Lima Sur & UNTELS & 1 \\
\hline Universidad Nacional de Barranca & UNAB & 4 \\
\hline Universidad Nacional de Juliaca & UNAJ & 3 \\
\hline Universidad Nacional de Frontera & UNF-S & 2 \\
\hline Universidad Nacional Autónoma de Alto Amazonas & UNAAA & 2 \\
\hline
\end{tabular}

* Clasificación con base en la Figura 1.

Fuente: SUNEDU (2016). 
institución, y la calidad educativa medida con una variable proxy, tal como es el puesto que ocupa la institución en el ranking de universidades elaborado por el CSIC (2019), el cual va de 1 a 25000 , en donde 1 es el valor más deseable. Con el fin de establecer relaciones positivas con la ETG, se optó por invertir los valores del ranking, aplicando la función inversa (1/ranking), luego se determinó el porcentaje de la participación relativa de cada universidad (índice de ranking mundial) en el total de los valores de la función inversa, lo cual garantiza que a mayor valor corresponde una mayor calidad de la institución educativa.

\section{Técnica de procesamiento y análisis de datos}

Se aplicó el DEA. Según Arieu (2004), dicho análisis permite la identificación del "mejor comportamiento", lo que posibilita el empleo de la técnica de benchmarking, a diferencia del análisis de regresión que se basa en el "comportamiento medio". Además de medir la eficiencia relativa, con el DEA se obtiene:

1. Una superficie envolvente empírica, que representa el comportamiento de los mejores.

2. Una métrica eficiente para comparar resultados.

3. Proyecciones eficientes sobre la frontera, para cada DMU ineficiente.

4. Un conjunto de referencia eficiente para cada DMU, definida por las unidades eficientes más próximas a ella. (Arieu, 2004, p. 3)

Se empleó el software SPSS Statistics 25 para el análisis descriptivo y correlacional, mientras que el software Frontier Analyst desarrollado por Banxia Software fue utilizado para el procesamiento de la data con el DEA.

\section{RESULTADOS}

\section{Estadísticos descriptivos de insumos y productos}

Debido a que la ETG presenta distribución normal $(p>0.05)$, mientras que los índices ETP y EE no presentan distribuciones normales $(p<0.05)$, se empleó el test $U$ de Mann-Whitney para comparar la hipótesis de que las distribuciones de los modelos RCE y RVE provienen de una misma población (Martín, 2006; Martín, 2008). Los resultados demuestran que efectivamente son iguales $(p>0.05)$, lo que evidencia que en el sector universitario de educación pública del Perú prevalecen los rendimientos constantes a escala. En la Tabla 2, se presentan los resultados de la ETG, los niveles actuales de insumos y productos, y el respectivo plan benchmarking de mejoras potenciales de producción para 42 universidades públicas peruanas, así como las variables que posteriormente serán correlacionadas con la ETG.

En la Figura 1, se presenta la matriz de orientación estratégica a la investigación y la masificación educativa (MOEIM) de 42 universidades públicas peruanas. Esta matriz se superpone a la gráfica de la frontera de producción de las universidades, que fue elaborada al dividir los productos entre el insumo, de tal manera que para cada DMU se estimaron 1. el número de investigadores Renacyt por cada 100 millones de soles de presupuesto (Renacyt_100MMSoles en el eje Y), y 2. la matrícula estudiantil por cada millón de soles de presupuesto (Matrícula/millón de soles en el eje X). Se observa que la línea de la frontera de producción (modelo orientado a las salidas) une las universidades eficientes (UNMSM, UNTELS y UNASAM), mientras que las universidades ineficientes se ubican por debajo de esta frontera.

La MOEIM ha sido segmentada en cuatro cuadrantes con base en los valores promedios de cada variable analizada (ver Anexo 1), de manera que en el caso de la variable investigadores Renacyt, se considera que la institución posee una alta orientación a la investigación (indicio de calidad de la investigación) cuando el valor promedio se encuentra por encima de la media y, por el contrario, baja cuando se encuentra por debajo de la media. En el caso de la variable matrícula, se considera que la institución posee una alta orientación a la masificación educativa (alto nivel de matrícula estudiantil) cuando el valor promedio se encuentra por encima de la media y, por el contrario, baja cuando se encuentra por debajo de la media, aunque también pudiera categorizarse como alta y baja orientación a la enseñanza, tal como se ha hecho en otros países (Shamohammadi y Oh, 2019).

En el primer cuadrante (superior derecho), se ubican las instituciones con orientación a la investigación y a la masificación, lideradas por la UNTELS. En el segundo cuadrante (superior izquierdo), se ubican las universidades con alta orientación a la investigación, encabezadas por la UNMSM y la UNALM con los niveles más altos de todas las instituciones; además, se ubican también las de baja orientación a la masificación. En el tercer cuadrante (inferior izquierdo), se ubican las universidades con baja orientación tanto a la investigación como a la 
Tabla 2. Eficiencia, niveles actuales y mejoras potenciales de producción para 42 universidades públicas peruanas y variables a correlacionar con la ETG.

\begin{tabular}{|c|c|c|c|c|c|c|c|c|}
\hline \multirow[b]{2}{*}{ SIGLAS } & \multirow[b]{2}{*}{ ETG (\%) } & \multicolumn{3}{|c|}{ Niveles actuales insumos y productos } & \multicolumn{2}{|c|}{ Metas (incrementos) } & \multicolumn{2}{|c|}{ Variables a correlacionar } \\
\hline & & $\begin{array}{l}\text { Presupuesto } \\
\text { (MM S/) }\end{array}$ & $\begin{array}{l}\text { Renacyt } \\
\text { (No.) }\end{array}$ & $\begin{array}{l}\text { Matrícula } \\
\text { (No.) }\end{array}$ & $\begin{array}{l}\text { Renacyt } \\
\text { (No.) }\end{array}$ & $\begin{array}{l}\text { Matrícula } \\
\text { (No.) }\end{array}$ & $\begin{array}{l}\text { Antigüedad } \\
\text { (Años) }\end{array}$ & $\begin{array}{l}\text { Ranking } \\
\text { Mundial }\end{array}$ \\
\hline UNMSM & 100.0 & 418.7 & 280 & 41011 & 0 & 0 & 469 & 1470 \\
\hline UNALM & 92.8 & 130.6 & 81 & 7303 & 6 & 5487 & 118 & 2966 \\
\hline UNI & 44.1 & 252.8 & 70 & 12914 & 89 & 16353 & 144 & 3044 \\
\hline UNSAAC & 49.7 & 174.3 & 24 & 21988 & 24 & 22249 & 328 & 3432 \\
\hline UNT & 71.8 & 156.3 & 56 & 19215 & 22 & 7554 & 36 & 3631 \\
\hline UNSA & 73.2 & 189.2 & 58 & 28520 & 21 & 10457 & 192 & 4623 \\
\hline UNASAM & 100.0 & 49.1 & 6 & 13603 & 0 & 0 & 43 & 5982 \\
\hline UNA & 60.2 & 177.3 & 42 & 23175 & 28 & 15314 & 164 & 6125 \\
\hline UNAP & 57.6 & 77.0 & 17 & 9819 & 13 & 7233 & 59 & 6225 \\
\hline UNPRG & 54.7 & 109.6 & 6 & 16618 & 7 & 13743 & 50 & 6647 \\
\hline UNFV & 58.9 & 154.5 & 1 & 25201 & 18 & 17599 & 57 & 6918 \\
\hline UNAC & 76.5 & 79.9 & 4 & 16921 & 6 & 5200 & 54 & 7027 \\
\hline UNHEVAL & 62.7 & 68.2 & 0 & 11838 & 8 & 7042 & 56 & 7585 \\
\hline UNJBG & 39.1 & 74.5 & 8 & 7401 & 12 & 11524 & 49 & 8013 \\
\hline UNC & 46.3 & 77.4 & 8 & 9381 & 9 & 10896 & 58 & 8109 \\
\hline UNP & 49.3 & 145.8 & 6 & 19933 & 12 & 20466 & 59 & 8642 \\
\hline UNACH & 30.7 & 16.2 & 1 & 1319 & 2 & 2981 & 10 & 9036 \\
\hline UNSM & 37.0 & 61.6 & 4 & 6138 & 7 & 10448 & 41 & 10319 \\
\hline UNSCH & 61.3 & 74.9 & 6 & 12649 & 4 & 7993 & 343 & 11088 \\
\hline UNAS & 41.0 & 52.6 & 10 & 4033 & 14 & 5796 & 56 & 11590 \\
\hline UNDAC & 47.6 & 66.9 & 1 & 8825 & 7 & 9716 & 55 & 11967 \\
\hline UNTumbes & 41.8 & 44.9 & 9 & 3372 & 13 & 4699 & 36 & 3631 \\
\hline UNCP & 91.3 & 99.9 & 13 & 24977 & 1 & 2391 & 58 & 8109 \\
\hline UNICA & 42.8 & 118.3 & 4 & 14045 & 10 & 18737 & 65 & 12578 \\
\hline UNH & 44.0 & 59.4 & 6 & 6818 & 8 & 8671 & 30 & 12595 \\
\hline UNE & 32.9 & 85.1 & 0 & 7753 & 10 & 15822 & 198 & 12865 \\
\hline UNFJFSC & 73.6 & 80.4 & 0 & 16390 & 10 & 5885 & 52 & 12965 \\
\hline UNAMBA & 86.4 & 21.5 & 9 & 3298 & 1 & 521 & 20 & 13566 \\
\hline UNTRM & 67.5 & 43.7 & 16 & 4501 & 8 & 2168 & 19 & 13617 \\
\hline UNS & 53.5 & 33.8 & 7 & 3971 & 6 & 3458 & 36 & 13653 \\
\hline UNU & 47.0 & 49.8 & 3 & 6462 & 3 & 7292 & 41 & 14293 \\
\hline UNAJMA & 33.4 & 15.3 & 1 & 1356 & 2 & 2702 & 16 & 15023 \\
\hline UNAMAD & 51.7 & 24.3 & 0 & 3475 & 3 & 3246 & 20 & 16366 \\
\hline UNAM & 24.8 & 29.4 & 3 & 1518 & 9 & 4612 & 15 & 16867 \\
\hline UNIA & 31.5 & 21.3 & 3 & 1299 & 7 & 2823 & 20 & 19332 \\
\hline UNDC & 46.4 & 9.0 & 1 & 1077 & 1 & 1243 & 11 & 20754 \\
\hline UNJ & 79.5 & 8.1 & 0 & 1783 & 1 & 459 & 12 & 21602 \\
\hline UNTELS & 100.0 & 12.3 & 4 & 3034 & 0 & 0 & 19 & 22067 \\
\hline UNAB & 74.2 & 8.9 & 0 & 1835 & 1 & 637 & 10 & 22078 \\
\hline UNAJ & 23.4 & 20.5 & 1 & 1263 & 3 & 4146 & 13 & 22095 \\
\hline UNF-S & 55.6 & 9.0 & 2 & 1062 & 2 & 848 & 10 & 23112 \\
\hline UNAAA & 28.3 & 5.4 & 1 & 157 & 3 & 399 & 13 & 24174 \\
\hline Totales & - & 3407.7 & 772 & 427251 & 411 & 298810 & - & - \\
\hline Media & 56.8 & 81.1 & 18 & 10173 & 10 & 7115 & - & - \\
\hline
\end{tabular}

ETG: eficiencia técnica global; MMS/.: millones de soles; No.: número de individuos Fuente: Elaboración propia. 
masificación, que es la peor situación de los cuatro tipos de desempeño analizados. Finalmente, en el cuarto cuadrante (inferior derecho), se ubican las universidades con baja orientación a la investigación, pero alta orientación a la masificación, que son encabezadas por la UNASAM.

Con el test de Shapiro-Wilk se corroboró estadísticamente que las variables experiencia (antigüedad) y calidad educativa (índice de ranking) de la institución no presentan distribuciones normales $(p<0.05)$, por lo cual se correlacionaron con la ETG mediante el coeficiente Rho de Spearman
(Tabla 3). Los resultados indican que la ET de las universidades públicas del Perú no se relaciona con la experiencia ni con la calidad educativa.

\section{DISCUSIÓN}

El estudio del sector educativo y, en especial, de la educación superior, es de suma importancia, dado que se existen lineamientos de política pública destinados a la mejora de la educación superior elaborados por instituciones internacionales y nacionales de prestigio como UNESCO, UNICEF, el Banco Mundial, UNFPA, PNUD (2015) y SUNEDU (Arias,

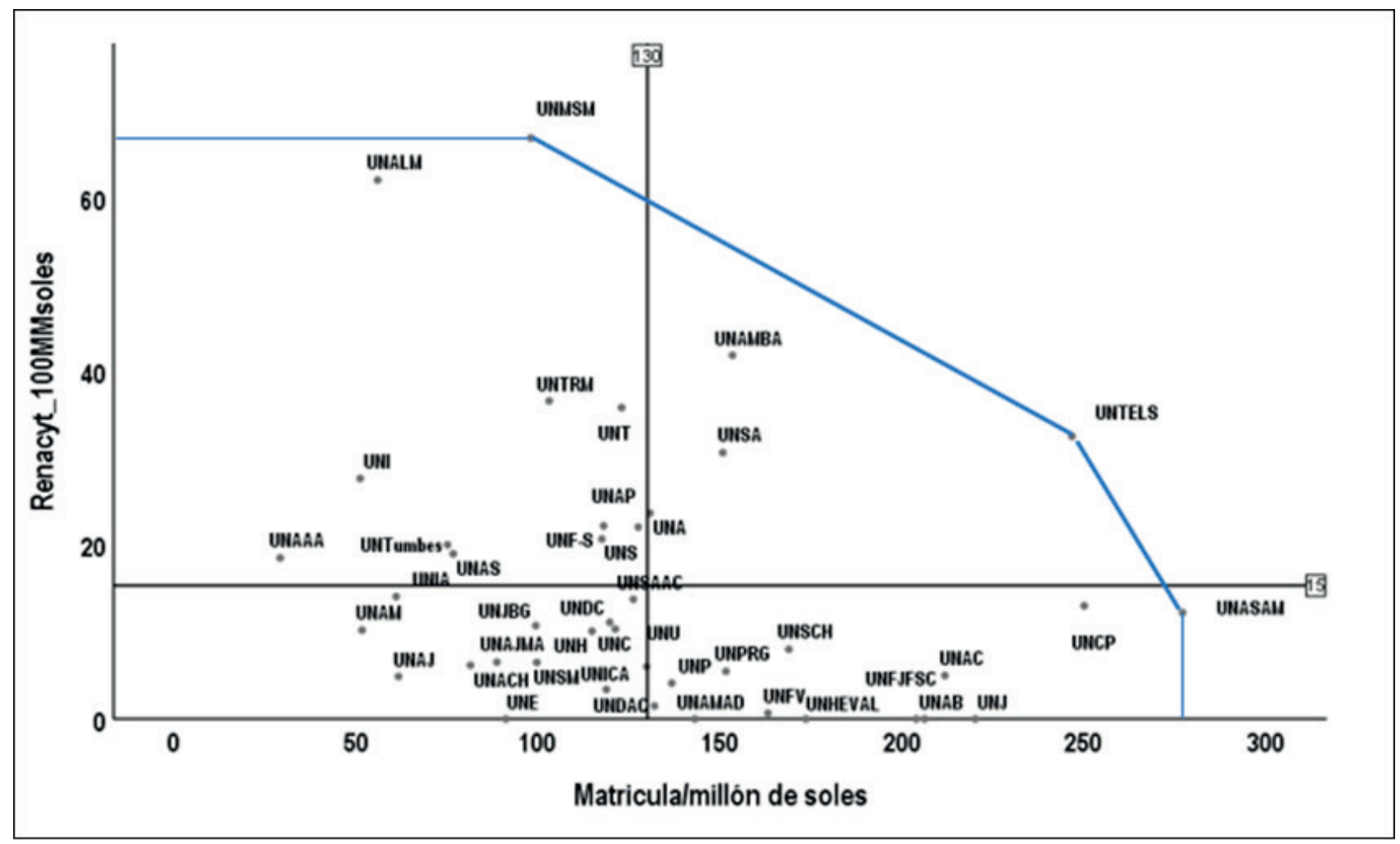

Figura 1. Matriz de orientación estratégica a la investigación y masificación educativa (MOEIM) de 42 universidades públicas peruanas.

Nota: La línea que une las tres universidades eficientes representa la frontera de producción.

Fuente: Elaboración propia.

Tabla 3. Correlaciones Rho de Spearman de la experiencia y calidad educativa con la ETG.

\begin{tabular}{|l|c|c|}
\hline Variables relacionadas con la ETG & Rho y significación & ETG \\
\hline \multirow{2}{*}{ Experiencia (antigüedad de la institución) } & Coeficiente de correlación & 0.232 \\
\cline { 2 - 3 } & Sig. (bilateral) & 0.140 \\
\hline \multirow{2}{*}{ Calidad educativa (ranking de universidades) } & Coeficiente de correlación & 0.298 \\
\cline { 2 - 3 } & Sig. (bilateral) & 0.055 \\
\hline
\end{tabular}

Fuente: Elaboración propia. 
2019), por citar algunas. La concreción de estos lineamientos permitiría aprovechar las grandes ventajas comparativas y competitivas, así como lograr el desarrollo sostenible en el Perú.

Uno de los principales problemas al momento de analizar la eficiencia en universidades es la existencia de una considerable variedad de insumos y productos en la literatura (Huamaní et al., 2016; Nieto, 2016; Blanco et al., 2019; Shamohammadi y Oh, 2019; Mojahedian et al., 2020), además de las dificultades para la medición de los mismos en algunos casos (Gómez, 2010; Ayaviri y Zamora, 2016; Buitrago et al., 2017). Por ello, se optó por emplear el modelo de Ramírez y Alfaro (2013), ligeramente modificado, para medir la función de investigación, cuyo modelo original fue utilizado exitosamente en 25 universidades chilenas. En dicho modelo, otros insumos como los docentes, estimado por la partida de sus remuneraciones, tienen una alta correlación con el presupuesto, lo cual desaconseja su utilización, pues es muy poco lo que aporta a los resultados. Asimismo, el modelo se desarrolló orientado a los productos, tal como se acostumbra en el sector universitario, debido a que, por lo general, los insumos no son controlados por las universidades estudiadas, lo que es más evidente en instituciones públicas cuyo objetivo principal es demostrar el mejor valor posible en los outputs al ser financiadas por el Estado (Buitrago et al., 2017).

Luego de justificar la orientación del modelo a emplear, se determinaron las fronteras de producción de los modelos de RCE y RVE, tal como se ha hecho en estudios previos (García y Palomares, 2008; Agasisti et al., 2011; Buitrago et al., 2017). Por otra parte, se aceptó la hipótesis según la cual las universidades públicas peruanas presentan funciones de producción de rendimientos constantes a escala. Para ello, en la presente investigación se demostró estadísticamente cuál de las fronteras de producción es la más apropiada, tal como lo hizo Martín (2008); en otros estudios sobre eficiencia se han calculado los dos tipos de frontera, sin demostrar estadísticamente cuál predomina (Haider et al., 2019). Esta demostración se realizó con base en un test estadístico no paramétrico que permitió comprobar que prevalecen los RCE, modelo que ha sido empleado por otros autores (Pino et al., 2010).

Bajo el enfoque RCE (índice ETG) y modelo orientado a los productos, se estimó que las universidades públicas del Perú tuvieron un 56.8\% de ET durante el año 2016. Este es un valor bajo en comparación con lo reportado por otros autores, aunque en contextos diferentes. Así, por ejemplo, Ramírez y Alfaro (2013) reportaron una eficiencia promedio de $80.89 \%$ en universidades públicas y privadas de Chile. Un valor muy cercano fue obtenido por Navarro et al. (2016) al aplicar un modelo DEA a un grupo de 32 universidades públicas de México y obtener una ETG de $80.7 \%$.

En el ámbito peruano, Nunez y Cornejo (2018), en un estudio de eficiencia de 42 universidades públicas y privadas, reportaron valores de eficiencia de $55.2 \%$ y $58.9 \%$, para las universidades públicas en dos de los cuatro modelos de DEA que evaluaron, cifras superiores a las reportadas para las instituciones privadas y que son muy similares a las obtenidas en la presente investigación, lo que confirma la existencia de un sector educativo muy heterogéneo.

Los resultados de la ET también permitieron elaborar una matriz que sirvió de base para clasificar a las instituciones en cuatro categorías según sus orientaciones estratégicas (alta o baja) hacia la investigación o masificación educativa. Esta dicotomía está muy presente en la educación superior, especialmente la latinoamericana (Cabrera et al., 2014; García de Fanelli, 2017) y, por ende, ha sido también reportada en el Perú (Lavalle y de Nicolas, 2017; Nunez y Cornejo, 2018).

Asimismo, se verificó la relación de la ET de las universidades públicas peruanas con la experiencia de la institución educativa (antigüedad), cuyo resultado obtenido al aplicar el test de correlación de Rho de Spearman indica que la ET de las universidades públicas peruanas no se relaciona con la experiencia, lo cual confirma los hallazgos de Coria (2019), quien no encontró relación entre la ET de las universidades argentinas de gestión estatal y la antigüedad de las mismas.

Igualmente, se relacionó la ET de las universidades públicas peruanas con la calidad de la institución educativa, medida con el ranking de universidades elaborado por el CSIC (2019). El coeficiente Rho de Spearman permite afirmar que la ET de las universidades públicas peruanas no se relaciona con la calidad de la institución educativa. Este resultado no coincide con los de otros investigadores que sí reportaron una relación positiva entre la eficiencia y los rankings de universidades, entre ellos el ranking de Shangai (Blanco et al., 2019), quizás por el hecho de que evaluaron a las 50 mejores universidades del mundo. Asimismo, Huamaní et al. (2016) demuestran la factibilidad de esta relación, ya que el lugar que se ocupa en los rankings es un sólido indicador de calidad educativa (Lavalle y de Nicolas, 2017). 
Finalmente, se propuso un plan de mejoras potenciales (plan benchmarking) con base en la metodología DEA, el cual puede ser aplicado como una herramienta de benchmarking (Avkiran, 1999; Zhu, 2009, p. 131), particularmente en el caso de las universidades (Shamohammadi y Oh, 2019). Este plan fue suficientemente demostrado, pues se pudo identificar las universidades eficientes que funcionarían como líderes del sector (benchmarks) para las universidades ineficientes.

\section{CONCLUSIONES}

- Las universidades públicas peruanas presentan funciones de producción de desempeño constante a escala (ETG promedio $=56.8 \%$, lo que sirvió de base para clasificar a las instituciones en cuatro categorías según sus orientaciones estratégicas (alta o baja) hacia la investigación o masificación educativa.

- La ET de las universidades públicas peruanas no está relacionada con la experiencia de la institución.

- La ET de las universidades públicas peruanas no está relacionada con la calidad de la educación.

- El acceso restringido a las publicaciones o patentes desarrolladas por las universidades se considera una limitación de este estudio, por lo cual deben abordarse en futuras investigaciones. Los datos solo están disponibles en los portales institucionales. También se puede incluir más insumos y productos, a fin de comparar sus resultados con los del presente modelo de pocas variables $\mathrm{y}$, adicionalmente, es relevante comparar la educación pública con la privada.

\section{REFERENCIAS BIBLIOGRÁFICAS}

[1] Abdurakhmanova, G., Shayusupova, N., Irmatova, A., y Rustamov, D. (2020). The Role of the Digital Economy in the Development of the Human Capital Market. International Journal of Psychological Rehabilitation, 24(07), 8043-8051.

[2] Agasisti, T., Dal Bianco, A., Landoni, P., Sala, A., y Salerno, M. (2011). Evaluating the Efficiency of Research in Academic Departments: An Empirical Analysis in an Italian Region. Higher Education Quarterly, 65(3), 267-289. Recuperado de https://doi.org/10.1111/j.14682273.2011.00489.x
[3] Arieu, A. (2004). Eficiencia técnica comparada en elevadores de granos de Argentina, bajo una aplicación de análisis de envolvente de datos. La situación del puerto de Bahía Blanca. Buenos Aires: Asociación Argentina de Economía Política. Recuperado de https:// aaep.org.ar/anales/works/works2004/Arieu.pdf

[4] Arias, P. (2019). La investigación: requisito para el avance de la calidad en las universidades peruanas. In Crescendo, 10(3), 447.

[5] Avkiran, N. (1999). An Application Reference for Data Envelopment Analysis in Branch Banking: Helping the Novice Researcher. International Journal of Bank Marketing, 17(5), 206-220. Recuperado de https://doi. org/10.1108/02652329910292675

[6] Ayaviri, V., y Zamora, G. (2016). Medición de la eficiencia en las universidades. Una propuesta metodológica. Perspectivas, (37), 7-22.

[7] Banker, R., Charnes, A., y Cooper, W. (1984). Some models for estimating technical and scale inefficiencies in data envelopment analysis. Management Science, 30(9), 10781092. Recuperado de https://doi.org/10.1287/ mnsc.30.9.1078

[8] Blanco, M., Bares, L., y Hrynevych, O. (2019). Análisis de la eficiencia global de las 50 mejores universidades del mundo. Revista Espacios, 40(9), 30. Recuperado de https://www. revistaespacios.com/a19v40n09/19400930. $\mathrm{html}$

[9] Blecich, A. (2020). Factors Affecting Relative Efficiency of Higher Education Institutions of Economic Orientation. Management: Journal of Contemporary Management Issues, 25(1), 4567. Recuperado de https://doi.org/10.30924/ mjcmi.25.1.3

[10] Buckle, R., y Creedy, J. (2019). The Evolution of Research Quality in New Zealand Universities as Measured by the Performance-Based Research Fund Process, New Zealand Economic Papers, 53(2), 144-165. Recuperado de https://doi.org/1 0.1080/00779954.2018.1429486

[11] Buitrago O., Espitia A., y Molano, L. (2017). Análisis envolvente de datos para la medición de la eficiencia en instituciones de educación superior: una revisión del estado del arte. Revista Científica General José María Córdova, 15(19), 147-173.

[12] Cabrera, A., Pérez, P., y López, L. (2014). Evolución de perspectivas en el estudio de la retención universitaria en los EE. UU.: 
Bases conceptuales y puntos de inflexión. En P. Figuera (Ed.), Persistir con éxito en la universidad: de la investigación a la acción (págs. 15-40). Barcelona, España: Laertes.

[13] Campoverde, J., Romero, C., y Borenstein, D. (2019). Evaluación de eficiencia de cooperativas de ahorro y crédito en Ecuador: aplicación del modelo Análisis Envolvente de Datos DEA. Contaduría y administración, 64(1), 1-19.

[14] Castañeda, P. (2019). Modelo de medición de la productividad para fábricas de software. (Tesis doctoral). Universidad Nacional Mayor de San Marcos, Lima.

[15] Charnes, A., Cooper, W., Lewin, A., y Seiford, L. (1994). Data Envelopment Analysis: Theory, Methodology, and Application. Kluwer Academic Publishers, Norwell.

[16] Charnes, A., Cooper, W., y Rhodes, E. (1978). Measuring the efficiency of decision making units. European journal of operational research, 2(6), 429-444.

[17] Coelli, T., Prasada, D. y Battese, G. (1998). An Introduction to Productivity and Efficiency Analysis. Boston, Estados Unidos: Kluwer academic publishers.

[18] Coria, M. (2019). Eficiencia técnica de las universidades argentinas de gestión estatal. Ensayos de Política Económica, 1(5), 44-64.

[19] Consejo Superior de Investigaciones Científicas (2019). Ranking Web de Universidades. Recuperado de https://www.webometrics.info/ es

[20] Cooper, W., Seiford, L., y Tone, K. (2006). Introduction to Data Envelopment Analysis and its Uses: With DEA-Solver Software and References. Springer Science \& Business Media.

[21] Díaz, J. (2008). Educación superior en el Perú: tendencias de la demanda y la oferta. En M. Benavides (Ed.), Análisis de programas, procesos y resultados educativos en el Perú: contribuciones empíricas para el debate (págs. 83-129). Lima, Perú: GRADE.

[22] Escalona, L. (2013). Eficiencia técnica para las universidades públicas venezolanas a través del modelo de análisis de datos envolventes DEA. Revista Científica "Teorías, Enfoques y Aplicaciones en las Ciencias Sociales”, 5(12), 45-62.
[23] Farrell, M. (1957). The measurement of Productive efficiency. Journal of the Royal Statistical Society. Series A, General, 125, 252267.

[24] García, A., y Palomares, D., (24-26 de setiembre de 2008). Evaluation of Spanish Universities: Efficiency, Technology and Productivity Change [artículo académico]. Prime-Latin America Conference, México D. F., México.

[25] García de Fanelli, A. (2017). Políticas públicas ante la masificación de la educación universitaria: el reto de elevar la graduación, garantizando la inclusión y la calidad. En C. Marquis (Ed.), La agenda universitaria III. Propuestas de políticas y acciones (págs. 167201). Buenos Aires, Argentina: Universidad de Palermo.

[26] Barbosa, S. (2010). Evaluación de la eficiencia de las escuelas de la Universidad Industrial de Santander aplicando análisis envolvente de datos (DEA). (Tesis doctoral). Universidad Industrial de Santander, Bucaramanga.

[27] Haider, M., Raza, Q., Jameel, S., y Pervaiz, K. (2019). A Comparative Study of Operational Efficiency of Pakistani and Malaysian Islamic Banks: Data Envelopment Analysis Approach. Asian Economic and Financial Review, 9(5), 559-580.

[28] Huamaní, G., Huamaní, S., Salcedo, J., y Fernández, C. (2016). Modelo de Análisis Envolvente de Datos (DEA) para evaluar la eficiencia de las escuelas profesionales de Ingeniería Industrial en el Perú con enfoque de desarrollo sostenible. Tecnia, 26(2), 2-72.

[29] Jiang, J., Lee, S., y Rah, M. (2020). Assessing the Research Efficiency of Chinese Higher Education Institutions by Data Envelopment Analysis. Asia Pacific Education Review, 21(3), 423-440.

[30] Kumar, A., y Thakur, R. (2019). Objectivity in Performance Ranking of Higher Education Institutions Using Dynamic Data Envelopment Analysis. International Journal of Productivity and Performance Management, 68(4), 774796.

[31] Lavalle, C., y de Nicolas, V. L. (2017). Peru and its New Challenge in Higher Education: Towards a Research University. PloS one, 12(8). Recuperado de https://doi.org/10.1371/ journal.pone.0182631

[32] Macilwain, C. (2010). Science Economics: What Science is Really Worth. Nature, 
465(7299), 682-684. Recuperado de https:// doi.org/10.1038/465682a

[33] Martín, R. (2006). La evaluación de la eficiencia técnica. Una aplicación del DEA a la Universidad de la Laguna [artículo científico]. XV Jornadas de la Asociación de la Economía de la Educación. Granada, España.

[34] Martín, R. (2008). La medición de la eficiencia universitaria: una aplicación del análisis envolvente de datos. Formación Universitaria, 1(2), 17-26. Recuperado de http://dx.doi. org/10.4067/S0718-50062008000200004

[35] Martin, J., y Roman, C. (2010). Evaluating the Service Quality of Major Air Carriers: A DEA Approach. International Journal of Applied Management Science, 2(4), 351-371.

[36] Mojahedian, M., Mohammadi, A., Abdollahi, M., Kebriaeezadeh, A., Sharifzadeh, M., Asadzandi, S., y Nikfar, S. (2020). A Review on Inputs and Outputs in Determining the Efficiency of Universities of Medical Sciences by Data Envelopment Analysis Method. Medical Journal of the Islamic Republic of Iran (MJIRI), 34(1), 34-42. Recuperado de http://mjiri.iums. ac.ir/article-1-6288-en.html

[37] Navarro, J., Gómez, R., y Torres, Z. (2016). Las universidades en México: una medida de su eficiencia a través del análisis de la envolvente de datos con bootstrap. Acta universitaria, 26(6), 60-69. Recuperado de https://www. redalyc.org/jatsRepo/416/41649084008/html/ index.html

[38] Nieto, L. (2016). Eficiencia y financiación en las universidades públicas españolas. (Tesis doctoral). Universidad Católica San Antonio de Murcia, Murcia.

[39] Nunez, N., y Cornejo, G. (2018). Haciendo mucho con poco: eficiencia de la investigación científica en el Perú. Revista Espacios, 39 (26), 1-7. Recuperado de http://www.revistaespacios. com/a18v39n26/a18v39n26p07.pdf

[40] Pino, J., Solís, M., Delgado, M., y Barea, R., (2010). Evaluación de la eficiencia de grupos de investigación mediante análisis envolvente de datos (DEA). El profesional de la información, 19(2), 160-167. Recuperado de https://doi. org/10.3145/epi.2010.mar.06

[41] Ramírez, P., y Alfaro, J. (2013). Evaluación de la eficiencia de las universidades pertenecientes al consejo de rectores de las universidades chilenas: Resultados de un análisis envolvente de datos. Formación universitaria, 6(3), 31-
38. Recuperado de http://dx.doi.org/10.4067/ S0718-50062013000300005

[42] Rhodes, E. (1978). Data envelopment analysis and related approaches for measuring the efficiency of decision-making unit with application to Program follow through U.S. education. (Tesis doctoral). Carnegie-Mellon University School of Urban and Public Affair, Pittsburgh.

[43] Rojas, M. (2010). Clasificación de los grupos de investigación de la facultad de Ingeniería de la Universidad Nacional de Colombia, mediante la estimación de la eficiencia técnica utilizando análisis envolvente de datos. (Trabajo de grado de maestría). Universidad Nacional de Colombia, Bogotá. Recuperado de https://repositorio.unal.edu.co/ bitstream/handle/unal/7466/02-822021.2010. pdf? sequence $=1$ \&isAllowed=y

[44] Salas-Velasco, M. (2020). The Technical Efficiency Performance of The Higher Education Systems Based on Data Envelopment Analysis with an Illustration for the Spanish Case. Educational Research for Policy and Practice, 19(2), 159-180. Recuperado de https://doi. org/10.1007/s10671-019-09254-5

[45] Schwab, K. (Ed.) (2019). The Global Competitive Report 2019. World Economic Forum. Recuperado de http://www3.weforum.org/docs/ WEF_TheGlobalCompetitivenessReport2019. pdf

[46] Shamohammadi, M., y Oh, D.-H. (2019). Measuring the Efficiency Changes of Private Universities of Korea: A Two-Stage Network Data Envelopment Analysis. Technological Forecasting and Social Change, 148, 119730. Recuperado de https://doi.org/10.1016/j. techfore.2019.119730

[47] Sigler, L. (2004). Aplicación del Data Envelopment Analysis a la producción de investigación económica en la Ciudad de México: La eficiencia relativa del CIDE, COLMEX, IPN, UAM y UNAM (1990-2002) [Ponencia]. 4th International Symposium of Data Envelopment Analysis and Performance Management, Birmingham, England.

[48] SUNEDU (2018). Información estadística de universidades. Recuperado de https:// www.sunedu.gob.pe/informacion-estadisticauniversidades-licenciadas/

[49] Torres, M., Vásquez, C., Luna, M, Bucci, N., Viloria, A., y Cabrera, D. (2019). Clustering of 
Top 50 Latin American Universities in SIR, QS, ARWU, and Webometrics Rankings. Procedia Computer Science, 160, 467-472. Recuperado de https://doi.org/10.1016/j.procs.2019.11.063

[50] Torres, M., Vásquez, C., Viloria, A., Borrero, T., Varela, N., Cabrera, D., Gaitán, M., y Gutiérrez, J. (2018). Efficiency analysis of the visibility of Latin American Universities and their impact on the ranking web. En Y. Tan, Y. Shi, y Q. Tang (Eds.), Data Mining and Big Data International Conference on Data Mining and Big Data (págs. 235-243). Cham, Siuza: Springer. Recuperado de https://doi.org/10.1007/978-3-319-938035_22

[51] UNESCO, UNICEF, Banco Mundial, UNFPA, PNUD, O. M. y A. (2015). Declaración de Incheon y Marco de Acción para la realización del Objetivo del Desarrollo Sostenible 4. Unescodoc, 1-84. Recuperado de https://unesdoc.unesco.org/ark:/48223/ pf0000245656_spa
[52] Wodon, Q. (2019). Measuring the Contribution of Faith-based Schools to Human Capital Wealth: Estimates for the Catholic Church. The Review of Faith \& International Affairs, 17(4), 94-102. Recuperado de https://doi.org/10.1080 /15570274.2019.1681782

[53] Zhao, Y. (2020). Transformation of Educational Management Mode in Ocean Colleges and Universities. Journal of Coastal Research, 110(1), 67-70. Recuperado de https://doi. org/10.2112/JCR-SI110-017.1

[54] Zhu, J. (2009). Quantitative Models for Performance Evaluation and Benchmarking. Data Envelopment Analysis with Spreadsheets ( $3^{\mathrm{a}}$ ed.). USA: Springer. 


\section{ANEXO}

Anexo 1. Caracterización de las universidades según resultados de la MOEIN.

\begin{tabular}{|lcccc|}
\hline \multicolumn{5}{|c}{ Universidades ubicadas en el segundo cuadrante } \\
\hline Siglas & $\begin{array}{c}\text { Presupuesto } \\
\text { (MM S/) }\end{array}$ & $\begin{array}{c}\text { Renacyt } \\
\text { (Nro.) }\end{array}$ & $\begin{array}{c}\text { Matrícula } \\
\text { (Nro.) }\end{array}$ & ETG \\
\hline UNAP & 77.0 & 17 & 9819 & 57.6 \\
UNT & 156.3 & 56 & 19215 & 71.8 \\
UNF-S & 9.0 & 2 & 1062 & 55.6 \\
UNS & 33.8 & 7 & 3971 & 53.5 \\
UNTRM & 43.7 & 16 & 4501 & 67.5 \\
UNMSM & 418.7 & 280 & 41011 & 100.0 \\
UNAS & 52.6 & 10 & 4033 & 41.0 \\
UNTumbes & 44.9 & 9 & 3372 & 41.8 \\
UNALM & 130.6 & 81 & 7303 & 92.8 \\
UNI & 252.8 & 70 & 12914 & 44.1 \\
UNAAA & 5.4 & 1 & 157 & 28.3 \\
\hline Promedio & 111.3 & 50 & 9760 & \\
\hline Total & 1224.8 & 549 & 107358 & \\
\hline \% del global & $35.9 \%$ & $71.1 \%$ & $25.1 \%$ & \\
\hline
\end{tabular}

\begin{tabular}{|lcccc|}
\hline \multicolumn{5}{|c|}{ Universidades ubicadas en el primer cuadrante } \\
\hline Siglas & $\begin{array}{c}\text { Presupuesto } \\
\text { (MM S/) }\end{array}$ & $\begin{array}{c}\text { Renacyt } \\
\text { (Nro.) }\end{array}$ & $\begin{array}{c}\text { Matrícula } \\
\text { (Nro.) }\end{array}$ & ETG \\
\hline UNTELS & 12.3 & 4 & 3034 & 100,0 \\
UNAMBA & 21.5 & 9 & 3298 & 86.4 \\
UNSA & 189.2 & 58 & 28520 & 73.2 \\
UNA & 177.3 & 42 & 23175 & 60.2 \\
\hline Promedio & 100.1 & 28 & 14507 & \\
\hline Total & 400.3 & 113 & 58027 & \\
\hline \% del global & $11.7 \%$ & $14.6 \%$ & $13.6 \%$ \\
\hline
\end{tabular}

\begin{tabular}{|lcccc|}
\hline \multicolumn{5}{c}{ Universidades ubicadas en el tercer cuadrante } \\
\hline \multicolumn{1}{|c}{ Siglas } & $\begin{array}{c}\text { Presupuesto } \\
\text { (MM S/) }\end{array}$ & $\begin{array}{c}\text { Renacyt } \\
\text { (Nro.) }\end{array}$ & $\begin{array}{c}\text { Matrícula } \\
\text { (Nro.) }\end{array}$ & ETG \\
\hline UNIA & 21.3 & 3 & 1299 & 31.5 \\
UNSAAC & 174.3 & 24 & 21988 & 49.7 \\
UNDC & 9.0 & 1 & 1077 & 46.4 \\
UNJBG & 74.5 & 8 & 7401 & 39.1 \\
UNC & 77.4 & 8 & 9381 & 46.3 \\
UNAM & 29.4 & 3 & 1518 & 24.8 \\
UNH & 59.4 & 6 & 6818 & 44.0 \\
UNAJMA & 15.3 & 1 & 1356 & 33.4 \\
UNSM & 61.6 & 4 & 6138 & 37.0 \\
UNACH & 16.2 & 1 & 1319 & 30.7 \\
UNU & 49.8 & 3 & 6462 & 47.0 \\
UNAJ & 20.5 & 1 & 1263 & 23.4 \\
UNICA & 118.3 & 4 & 14045 & 42.8 \\
UNE & 85.1 & 0 & 7753 & 32.9 \\
\hline Promedio & 58.0 & 5 & 6273 & \\
\hline Total & 812.1 & 67 & 87818 & \\
\hline \% del global & $23.8 \%$ & $8.7 \%$ & $20.6 \%$ & \\
\hline
\end{tabular}

\begin{tabular}{|lcccc|}
\hline \multicolumn{5}{|c|}{ Universidades ubicadas en el cuarto cuadrante } \\
\hline \multicolumn{1}{|c}{ Siglas } & $\begin{array}{c}\text { Presupuesto } \\
\text { (MM S/) }\end{array}$ & $\begin{array}{c}\text { Renacyt } \\
\text { (Nro.) }\end{array}$ & $\begin{array}{c}\text { Matrícula } \\
\text { (Nro.) }\end{array}$ & ETG \\
\hline UNCP & 99.9 & 13 & 24977 & 91.3 \\
UNASAM & 49.1 & 6 & 13603 & 100.0 \\
UNSCH & 74.9 & 6 & 12649 & 61.3 \\
UNPRG & 109.6 & 6 & 16618 & 54.7 \\
UNAC & 79.9 & 4 & 16921 & 76.5 \\
UNP & 145.8 & 6 & 19933 & 49.3 \\
UNDAC & 66.9 & 1 & 8825 & 47.6 \\
UNFV & 154.5 & 1 & 25201 & 58.9 \\
UNJ & 8.1 & 0 & 1783 & 79.5 \\
UNAB & 8.9 & 0 & 1835 & 74.2 \\
UNFJFSC & 80.4 & 0 & 16390 & 73.6 \\
UNHEVAL & 68.2 & 0 & 11838 & 62.7 \\
UNAMAD & 24.3 & 0 & 3475 & 51.7 \\
\hline Promedio & 74.7 & 3 & 13388 & \\
\hline Total & 970.5 & 43 & 174048 \\
\hline \% del global & $28.5 \%$ & $5.6 \%$ & $40.7 \%$ \\
\hline
\end{tabular}

Total de universidades (insumos y productos)

\begin{tabular}{|lccc|}
\hline & $\begin{array}{l}\text { Presupuesto } \\
\text { (MM S/) }\end{array}$ & $\begin{array}{l}\text { Renacyt } \\
\text { (Nro.) }\end{array}$ & $\begin{array}{l}\text { Matrícula } \\
\text { (Nro.) }\end{array}$ \\
\hline Total & 3407.7 & 772 & 427251 \\
\hline$\%$ & $100.0 \%$ & $100.0 \%$ & $100.0 \%$ \\
\hline
\end{tabular}




\title{
Technical Efficiency of Public Universities in Peru
}

\author{
Marco Antonio Tello Miranda \\ JosÉ OVIDIO FLORES GUTIÉRREZ 2
}

\begin{abstract}
Deficiencies in the quality of higher education are among Peru's main weaknesses according to the world competitiveness ranking. Therefore, this study determined the technical efficiency (TE) of Peruvian public universities using data envelopment analysis (DEA). In addition, it analyzed the relationships between the experience and education quality of the institutions, and TE. The study covered the 42 public universities registered for 2016, which were measured in terms of one input and two outputs. It was concluded that Peruvian public universities have production functions of constant returns to scale (average OTE $=56.8 \%$ ), which served as a basis for classifying institutions into four categories according to their strategic orientations (high or low) towards research or universal education. It was also found that no relationship exists between TE and the experience or education quality of the institution.
\end{abstract}

Keywords: university; data envelopment analysis (DEA); benchmarking; quality; experience.

\section{INTRODUCTION}

A number of economic theories strongly maintain that the future of a country's economy depends on the investment in and development of education, science and technology (Abdurakhmanova et al., 2020; Macilwain, 2010), which implies that education is a significant variable in the development of any nation, since human capital is crucial for socio-economic growth and, hence, for sustainable development (Wodon, 2019; Blecich, 2020). Therefore, a country's education policy must prioritize the enhancement of education quality in all its branches, coordinating public policies to optimize infrastructure, as well as the quality of teacher training and performance, among other important goals (Buckle \& Creedy, 2019; Zhao, 2020).

Measuring and improving the efficiency of university performance is considered a relevant topic in developed countries (Kumar \& Thakur, 2019; Jiang et al., 2020) and of special interest in public management (Ayaviri \& Zamora, 2016). Similarly, public universities in Latin American countries acknowledge the need for changes and adaptation to new trends in higher education worldwide, which increasingly demand greater efficiency and quality in the services offered (Torres et al., 2019).

According to the World Competitiveness Ranking 2019, Peru ranked 65th and its main weaknesses remain in the following pillars: institutions, unlicensed universities, infrastructure, education, and innovation capability, among others (Schwab, 2019). Several authors have also reported deficiencies in Peruvian higher education (Lavalle \& de Nicolas, 2017; Nunez \& Cornejo, 2018), therefore, this research aims to determine technical efficiency (TE) in Peruvian public universities by means of the mathematical technique known as Data Envelopment Analysis (DEA), used to measure and evaluate the results obtained in productive

\footnotetext{
Degree in Chemical Engineering from Universidad Nacional Mayor de San Marcos (Lima, Peru). Currently working as teacher at the CERSEU of the School of Industrial Engineering of UNMSM. (Lima, Peru).

ORCID: https://orcid.org/0000-0002-4759-5772

Corresponding author: marco.tello@unmsm.edu.pe

$2 \mathrm{PhD}$ in Engineering from Universidad de Valladolid (Spain). Currently working as postgraduate professor at Universidad Nacional Mayor de San Marcos (Lima, Peru). ORCID: https://orcid.org/0000-0001-5019-2635

E-mail: floresjoseovidio@gmail.com
} 
units by comparing their productivity levels (Sigler, 2004; Rojas, 2010; Torres et al., 2018). Additionally, the existing relationships between the experience and education quality of the institutions, and technical efficiency are determined.

Higher education is of vital importance for the sustainable development of countries, and therefore the efficiency of its system should be considered a priority. In this context, this research aims to determine the technical efficiency (TE) of public universities in Peru; in addition, it intends to analyze the relationship between ET and the experience and education quality of the institutions.

This research study introduces a DEA model with a single input and two outputs that represent the relevant variables for the system analysis, which is particularly useful in contexts of scarce or limited information - very common in developing countries-. An easy-to-use, simple tool called Matrix of strategic orientation towards research and universal education (MOEIM, by its Spanish acronym) is elaborated based on the results of the DEA model, providing a comprehensive view of the positioning of universities in terms of their relative efficiency. The MOEIM provides a comprehensive overview of the positioning of universities in terms of their relative efficiency, which is relevant for guiding decision-making in universities and in the institutions that design and implement public policies focused on higher education. This tool is complemented by the benchmarking plan based on the DEA technique.

\section{Data Envelopment Analysis (DEA)}

According to Buitrago et al. (2017), DEA is a technique used to measure relative efficiency in educational organizations; it is important to note that it was first used to measure educational efficiency in North American schools (Rhodes, 1978). Its accuracy in measuring the decision-making unit under evaluation, as well as its inputs and outputs, stands out among its strengths; accordingly, following application of the technique, efficient and inefficient units can be classified, and reference pairs can be assigned to provide guidelines for improving inefficient units and establishing goals for the correct use of resources.

Based on a study on public universities in Spain by Salas-Velasco (2020), DEA is a good instrument for the comparative evaluation of higher education. In our context, by using inputs and outputs, DEA can identify technically efficient institutions that may act as benchmarks for ranking universities; in addition, it allows to identify the factors negatively impacting technical efficiency of the universities under evaluation.

Castañeda (2019) states that DEA is a widespread mathematical programming technique devised by Charnes et al. (1978) and improved by Banker et al. (1984) to evaluate variable returns to scale. DEA generalizes Farrell (1957) single-output/input technical efficiency measure to the multiple-output/ multiple-input cases (Charnes et al., 1994; Cooper et al., 2006). Organizations or units that are measured using DEA are referred to as "decision making units" (DMUs) and, according to Cooper et al. (2006), a DMU is considered efficient if no other DMU can produce more outputs using an equal or lesser amount of inputs.

Technical efficiency aims at maximizing the results of the DMU based on the resources used (Campoverde et al., 2019). Thus, a university is efficient when it manages to obtain maximum levels of outputs (or outputs) for a given level of inputs or, alternatively, when it is able to minimize the level of inputs for a given level of output. The main contribution of DEA consists of establishing, empirically or practically, a reference pattern via a production frontier, against which the DMU is compared to determine whether it is efficient or not. Thus a relative efficiency is established, where the estimation of the frontier serves to estimate ET. Farrell (1957) provided a method to calculate and classify efficiency into technical and allocative efficiency, formulating precepts on the constant returns to scale of technology and a convex isoquant to the origin that has a positive slope.

In summary, a production function, which indicates the maximum amount of output generated by each input, must be defined to perform an efficiency analysis. Additionally, the characteristics of the process and the sector to which the DMUs under analysis belong should be outlined.

To associate the production function with the variables that reflect technology and enable the most efficient production of DMUs, such characterization should include the technology applied in the production process, and the inputs and outputs of the system. This approach can be understood in relation to outputs or inputs, so that an output-oriented application (as in this research) would show the maximum production that can be achieved with a particular combination of factors; while an input-oriented analysis would reveal the minimum requirements of inputs, combined in a given proportion, to achieve a specific output level (Escalona, 2013). 
Technical efficiency can also be called overall technical efficiency (OTE) and it consists of pure technical efficiency (PTE) and scale efficiency (SE), so that OTE = PTE $x$ SE. Therefore, to determine whether the production technology applied by universities have variable returns to scale, two models are applied: constant returns to scale (CRS), which corresponds to the OTE frontier; and variable returns to scale (VRS), which corresponds to the PTE frontier (Blecich, 2020). Should differences be detected between the two measurements for a particular university, it is assumed that there are scale inefficiencies, which values are attributed to the discrepancy between the CRS and VRS measurements. Thus, the scale efficiency measure is obtained as a result of the quotient between the OTE measure and the PTE measure. In summary, PTE evaluates the university's technical efficiency as a specific result of the organization's management without considering the size of the organization (Martin and Roman, 2010), whereas scale inefficiencies are losses caused by failure to operate at the optimal production size. Then, it follows that a university can be technically efficient and still have the possibility to improve its performance if the decision is made to exploit economies of scale (Coelli et al., 1998, p. 4).

Therefore, those universities that achieve values of $100 \%$ (or 1 ) for a given type of performance (CRS or VRS) against which they are being compared will be efficient and, therefore, are considered to be above the production frontier or to be part of it. In contrast, values below $100 \%(100 \%>x \geq 0)$ denote inefficiencies and, as such, will be located below the production frontier.

\section{Peruvian Higher Education System}

Arias (2019) reported that towards the end of 2019, the Superintendencia Nacional de Educación Superior Universitaria (SUNEDU) ${ }^{3}$ should have completed the licensing process for the remaining Peruvian universities. Research is one of the indicators of education quality and is a requirement for university licensing. It must be conducted professionally at the universities and, given that there is little research, it constitutes the main difficulty encountered by some universities

In Peru, university education is not compulsory and, hence, represents an educational option for people who graduate from the basic education system and intend to pursue professional, artistic or technical studies (Díaz, 2008). Consequently, the demand for

3 Public body in charge of regulating higher education in Peru. university education can be estimated in terms of the number of people who, after completing secondary school, continue higher education by applying to any educational institution or, more restrictively, in terms of the number of individuals who apply and are admitted.

\section{METHODOLOGY}

This is a quantitative, applied research, with a descriptive and correlational scope, and a non-experimental and cross-sectional design. The sample comprised the 42 Peruvian public universities registered for 2016 (Table 1), details of which were obtained from each university's website and also provided by SUNEDU (2018).

Based on a model proposed by Ramírez and Alfaro (2013), slightly modified to measure the research function, the only input used was the budget in millions (MM) of soles of each university and two outputs: the number of researchers assigned to the Renacyt program (formerly Regina) and the number of undergraduate, master's and doctoral students enrolled. Other inputs, such as number of professors, have a high correlation with the budget in public universities. Additionally, it was not possible to specify the number of articles per university published in indexed journals because they are not available, as mentioned above.

Variables to be correlated with TE were seniority (years), representing the institution's experience, and educational quality measured with a proxy variable, such as the place occupied by the institution in the ranking of universities by the CSIC (2019), which ranges from 1 to 25000 , where 1 is the most desirable score. To establish positive relationships with the OTE, we inverted the ranking values, applying the inverse function (1/ranking), and then determined the percentage of the relative participation of each university (world ranking index) in the total values of the inverse function, which guarantees that the higher the value, the higher the quality of the educational institution.

\section{Data Processing and Analysis Technique}

DEA was applied. Arieu (2004) states that this analysis allows for the identification of the "best performance", thereby making it possible to use the benchmarking technique, as opposed to regression analysis, which is based on "average performance". In addition to measuring relative efficiency, DEA provides: 
Table 1. List of the 42 Peruvian Public Universities Under Study.

\begin{tabular}{|c|c|c|}
\hline University & Acronym & Quadrant (strategy matrix)* \\
\hline Universidad Nacional Mayor de San Marcos & UNMSM & 2 \\
\hline Universidad Nacional Agraria La Molina & UNALM & 2 \\
\hline Universidad Nacional de Ingeniería & UNI & 2 \\
\hline Universidad Nacional de San Antonio Abad del Cusco & UNSAAC & 3 \\
\hline Universidad Nacional de Trujillo & UNT & 2 \\
\hline Universidad Nacional de San Agustín & UNSA & 1 \\
\hline Universidad Nacional Santiago Antúnez de Mayolo & UNASAM & 4 \\
\hline Universidad Nacional del Altiplano & UNA & 1 \\
\hline Universidad Nacional de la Amazonía Peruana & UNAP & 2 \\
\hline Universidad Nacional Pedro Ruíz Gallo & UNPRG & 4 \\
\hline Universidad Nacional Federico Villarreal & UNFV & 4 \\
\hline Universidad Nacional del Callao & UNAC & 4 \\
\hline Universidad Nacional Hermilio Valdizán & UNHEVAL & 4 \\
\hline Universidad Nacional Jorge Basadre Grohmann & UNJBG & 3 \\
\hline Universidad Nacional de Cajamarca & UNC & 3 \\
\hline Universidad Nacional de Piura & UNP & 4 \\
\hline Universidad Nacional Autónoma de Chota & UNACH & 3 \\
\hline Universidad Nacional de San Martín & UNSM & 3 \\
\hline Universidad Nacional de San Cristóbal de Huamanga & UNSCH & 4 \\
\hline Universidad Nacional Agraria de la Selva & UNAS & 2 \\
\hline Universidad Nacional Daniel Alcídes Carrión & UNDAC & 4 \\
\hline Universidad Nacional de Tumbes & UNTumbes & 2 \\
\hline Universidad Nacional del Centro del Perú & UNCP & 4 \\
\hline Universidad Nacional San Luis Gonzaga & UNICA & 3 \\
\hline Universidad Nacional de Huancavelica & UNH & 3 \\
\hline Universidad Nacional de Educación Enrique Guzmán y Valle & UNE & 3 \\
\hline Universidad Nacional José Faustino Sánchez Carrión & UNFJFSC & 4 \\
\hline Universidad Nacional Micaela Bastidas de Apurímac & UNAMBA & 1 \\
\hline Universidad Nacional Toribio Rodríguez de Mendoza de Amazonas & UNTRM & 2 \\
\hline Universidad Nacional del Santa & UNS & 2 \\
\hline Universidad Nacional de Ucayali & UNU & 3 \\
\hline Universidad Nacional José María Arguedas & UNAJMA & 3 \\
\hline Universidad Nacional Amazónica de Madre de Dios & UNAMAD & 4 \\
\hline Universidad Nacional de Moquegua & UNAM & 3 \\
\hline Universidad Nacional Intercultural de la Amazonía & UNIA & 3 \\
\hline Universidad Nacional de Cañete & UNDC & 3 \\
\hline Universidad Nacional de Jaén & UNJ & 4 \\
\hline Universidad Nacional Tecnológica de Lima Sur & UNTELS & 1 \\
\hline Universidad Nacional de Barranca & UNAB & 4 \\
\hline Universidad Nacional de Juliaca & UNAJ & 3 \\
\hline Universidad Nacional de Frontera & UNF-S & 2 \\
\hline Universidad Nacional Autónoma de Alto Amazonas & UNAAA & 2 \\
\hline
\end{tabular}

* Classification based on Figure 1.

Source: SUNEDU (2016). 
1. An empirical envelope surface, which represents the behavior of the best performers

\section{An efficient metric to compare results.}

3. Efficient projections on the frontier for each inefficient DMU.

4. An efficient reference set for each DMU, defined by the efficient units closest to it. (Arieu, 2004, p. 3)

SPSS Statistics 25 software was used for descriptive and correlational analysis, while Frontier Analyst software developed by Banxia Software was used for data processing with the DEA.

\section{RESULTS}

\section{Descriptive Statistics for Inputs and Outputs}

As OTE has a normal distribution ( $p>0.05)$, whereas the PTE and EE values do not $(p<0.05)$, the Mann-Whitney $U$ test was used to contrast the hypothesis stating that the distributions of the CRS and VRS models come from the same population (Martín, 2006; Martín, 2008). Results show that they are indeed equal $(p>0.05)$, which is evidence that constant returns to scale prevail in the Peruvian public university education sector. Table 2 presents the results of the OTE, the current levels of inputs and outputs, and the respective benchmarking plan on potential output improvement for 42 Peruvian public universities, as well as the variables that will also be correlated with the OTE.

Figure 1 shows the Matrix of strategic orientation towards research and universal education (MOEIM, by its Spanish acronym) of 42 Peruvian public universities. This matrix overlaps the graph of the universities' production frontier, developed by dividing the outputs by the input, so that for each DMU we estimated 1. the number of Renacyt researchers per 100 million soles of budget (Renacyt_100MMSoles on the Y-axis), and 2. student enrollment per million soles of budget (Enrollment/million soles on the $\mathrm{X}$-axis). It is observed that the production frontier line (output-oriented model) links the efficient universities (UNMSM, UNTELS and UNASAM), while the inefficient universities are located below this frontier.

The MOEIM was divided into four quadrants based on the average values of each variable analyzed (see Appendix 1). Accordingly, in the case of the Renacyt researchers variable, the institution has a high research orientation (indicative of research quality) when the average value is above the mean and low orientation when it is below the mean. As for the case of the enrollment variable, the institution has a high orientation to universal education (high level of student enrollment) when the average value is above the mean and low when it is below the mean, although it could also be categorized as high and low orientation teaching orientation, as has been done in other countries (Shamohammadi \& Oh, 2019).

The first quadrant (upper right) includes research-oriented and universal education-oriented institutions, led by UNTELS. The second quadrant (upper left) includes universities with a high research orientation, with UNMSM and UNALM leading with the highest scores; also, those universities with a low orientation towards universal education are also located in this quadrant. The third quadrant (lower left) includes universities with a low orientation towards both research and universal education, which is the worst case among the four types of performance analyzed. Finally, the fourth quadrant (lower right) includes universities with a low orientation towards research but a high orientation towards universal education, led by UNASAM.

Shapiro-Wilk test statistically corroborated that the variables experience (seniority) and education quality (ranking) of the institution do not have normal distributions $(p<0.05)$, so they were correlated with OTE using Spearman's Rho coefficient (Table 3). Results indicate that the TE of public universities in Peru is not correlated to experience or education quality.

\section{DISCUSSION}

Research on the education sector and, particularly, on higher education, is of utmost importance in light of the existence of public policy guidelines aimed at improving higher education developed by prestigious international and national institutions such as UNESCO, UNICEF, the World Bank, UNFPA, UNDP (2015) and SUNEDU (Arias, 2019), to name a few. Putting these guidelines into practice would make it possible to capitalize on the great comparative and competitive advantages, as well as to achieve sustainable development in Peru.

Among the main problems encountered when analyzing efficiency in universities is the existence of a wide variety of inputs and outputs in the literature (Huamaní et al., 2016; Nieto, 2016; Blanco et al., 2019; Shamohammadi, \& Oh, 2019; Mojahedian et al., 2020), in addition to the difficulties in measuring 
Table 2. Efficiency, Current Levels and Potential Output Improvement for 42 Peruvian Public Universities and Variables to Correlate with the OTE.

\begin{tabular}{|c|c|c|c|c|c|c|c|c|}
\hline \multirow[b]{2}{*}{ ACRONYM } & \multirow[b]{2}{*}{ OTE (\%) } & \multicolumn{3}{|c|}{ Current Input and Output Levels } & \multicolumn{2}{|c|}{ Targets (growth) } & \multicolumn{2}{|c|}{ Variables to Correlate } \\
\hline & & $\begin{array}{l}\text { Budget } \\
\text { (MM S/) }\end{array}$ & $\begin{array}{l}\text { Renacyt } \\
\text { (No.) }\end{array}$ & $\begin{array}{l}\text { Enrollment } \\
\text { (No.) }\end{array}$ & $\begin{array}{l}\text { Renacyt } \\
\text { (No.) }\end{array}$ & $\begin{array}{l}\text { Enrollment } \\
\text { (No.) }\end{array}$ & $\begin{array}{l}\text { Seniority } \\
\text { (years) }\end{array}$ & $\begin{array}{l}\text { World } \\
\text { Ranking }\end{array}$ \\
\hline UNMSM & 100.0 & 418.7 & 280 & 41011 & 0 & 0 & 469 & 1470 \\
\hline UNALM & 92.8 & 130.6 & 81 & 7303 & 6 & 5487 & 118 & 2966 \\
\hline UNI & 44.1 & 252.8 & 70 & 12914 & 89 & 16353 & 144 & 3044 \\
\hline UNSAAC & 49.7 & 174.3 & 24 & 21988 & 24 & 22249 & 328 & 3432 \\
\hline UNT & 71.8 & 156.3 & 56 & 19215 & 22 & 7554 & 36 & 3631 \\
\hline UNSA & 73.2 & 189.2 & 58 & 28520 & 21 & 10457 & 192 & 4623 \\
\hline UNASAM & 100.0 & 49.1 & 6 & 13603 & 0 & 0 & 43 & 5982 \\
\hline UNA & 60.2 & 177.3 & 42 & 23175 & 28 & 15314 & 164 & 6125 \\
\hline UNAP & 57.6 & 77.0 & 17 & 9819 & 13 & 7233 & 59 & 6225 \\
\hline UNPRG & 54.7 & 109.6 & 6 & 16618 & 7 & 13743 & 50 & 6647 \\
\hline UNFV & 58.9 & 154.5 & 1 & 25201 & 18 & 17599 & 57 & 6918 \\
\hline UNAC & 76.5 & 79.9 & 4 & 16921 & 6 & 5200 & 54 & 7027 \\
\hline UNHEVAL & 62.7 & 68.2 & 0 & 11838 & 8 & 7042 & 56 & 7585 \\
\hline UNJBG & 39.1 & 74.5 & 8 & 7401 & 12 & 11524 & 49 & 8013 \\
\hline UNC & 46.3 & 77.4 & 8 & 9381 & 9 & 10896 & 58 & 8109 \\
\hline UNP & 49.3 & 145.8 & 6 & 19933 & 12 & 20466 & 59 & 8642 \\
\hline UNACH & 30.7 & 16.2 & 1 & 1319 & 2 & 2981 & 10 & 9036 \\
\hline UNSM & 37.0 & 61.6 & 4 & 6138 & 7 & 10448 & 41 & 10319 \\
\hline UNSCH & 61.3 & 74.9 & 6 & 12649 & 4 & 7993 & 343 & 11088 \\
\hline UNAS & 41.0 & 52.6 & 10 & 4033 & 14 & 5796 & 56 & 11590 \\
\hline UNDAC & 47.6 & 66.9 & 1 & 8825 & 7 & 9716 & 55 & 11967 \\
\hline UNTumbes & 41.8 & 44.9 & 9 & 3372 & 13 & 4699 & 36 & 3631 \\
\hline UNCP & 91.3 & 99.9 & 13 & 24977 & 1 & 2391 & 58 & 8109 \\
\hline UNICA & 42.8 & 118.3 & 4 & 14045 & 10 & 18737 & 65 & 12578 \\
\hline UNH & 44.0 & 59.4 & 6 & 6818 & 8 & 8671 & 30 & 12595 \\
\hline UNE & 32.9 & 85.1 & 0 & 7753 & 10 & 15822 & 198 & 12865 \\
\hline UNFJFSC & 73.6 & 80.4 & 0 & 16390 & 10 & 5885 & 52 & 12965 \\
\hline UNAMBA & 86.4 & 21.5 & 9 & 3298 & 1 & 521 & 20 & 13566 \\
\hline UNTRM & 67.5 & 43.7 & 16 & 4501 & 8 & 2168 & 19 & 13617 \\
\hline UNS & 53.5 & 33.8 & 7 & 3971 & 6 & 3458 & 36 & 13653 \\
\hline UNU & 47.0 & 49.8 & 3 & 6462 & 3 & 7292 & 41 & 14293 \\
\hline UNAJMA & 33.4 & 15.3 & 1 & 1356 & 2 & 2702 & 16 & 15023 \\
\hline UNAMAD & 51.7 & 24.3 & 0 & 3475 & 3 & 3246 & 20 & 16366 \\
\hline UNAM & 24.8 & 29.4 & 3 & 1518 & 9 & 4612 & 15 & 16867 \\
\hline UNIA & 31.5 & 21.3 & 3 & 1299 & 7 & 2823 & 20 & 19332 \\
\hline UNDC & 46.4 & 9.0 & 1 & 1077 & 1 & 1243 & 11 & 20754 \\
\hline UNJ & 79.5 & 8.1 & 0 & 1783 & 1 & 459 & 12 & 21602 \\
\hline UNTELS & 100.0 & 12.3 & 4 & 3034 & 0 & 0 & 19 & 22067 \\
\hline UNAB & 74.2 & 8.9 & 0 & 1835 & 1 & 637 & 10 & 22078 \\
\hline UNAJ & 23.4 & 20.5 & 1 & 1263 & 3 & 4146 & 13 & 22095 \\
\hline UNF-S & 55.6 & 9.0 & 2 & 1062 & 2 & 848 & 10 & 23112 \\
\hline UNAAA & 28.3 & 5.4 & 1 & 157 & 3 & 399 & 13 & 24174 \\
\hline Totales & - & 3407.7 & 772 & 427251 & 411 & 298810 & - & - \\
\hline Media & 56.8 & 81.1 & 18 & 10173 & 10 & 7115 & - & - \\
\hline
\end{tabular}

OET: overall technical efficiency; MMS/.: millions of soles; No.: number of individuals.

Source: Prepared by the authors. 


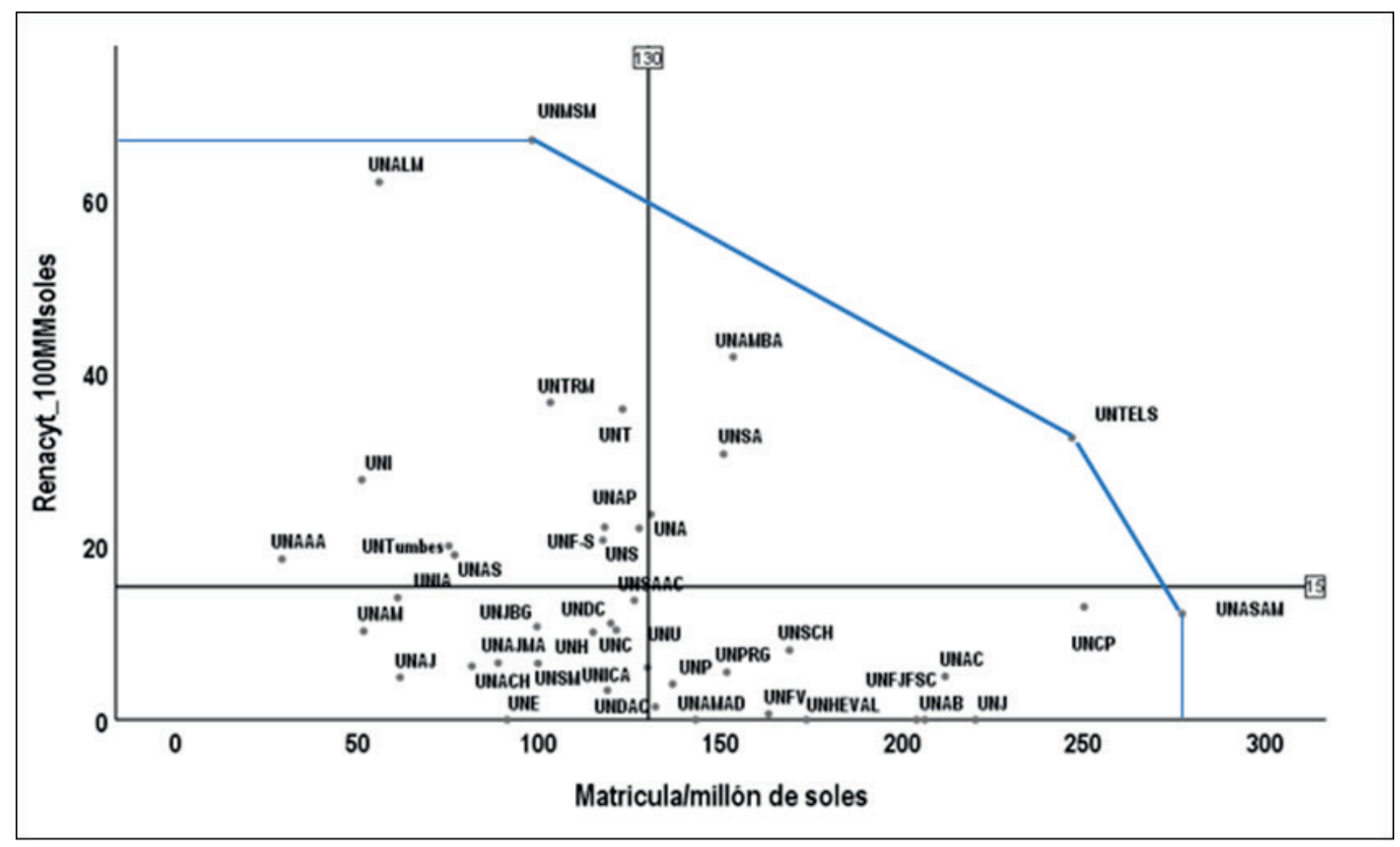

Figure 1. Matrix of strategic orientation towards research and universal education (MOEIM) of 42 Peruvian public universities.

Note: The line joining the three efficient universities represents the production frontier.

Source: Prepared by the authors.

Table 3. Spearman's Rho Correlations Between Experience and Education Quality and OTE

\begin{tabular}{|lll|}
\hline Variables related to the OTE & \multicolumn{1}{c|}{ Rho and Significance } & OTE \\
\hline \multirow{2}{*}{ Experience (seniority) } & Correlation Coefficient & 0.232 \\
\hline \multirow{2}{*}{ Education Quality (university ranking) } & Sig. (2-tailed) & 0.140 \\
\hline
\end{tabular}

Source: Prepared by the authors.

them in some cases (Gómez, 2010; Ayaviri \& Zamora, 2016; Buitrago et al., 2017). It was therefore decided to use the model of Ramírez and Alfaro (2013), slightly modified, to measure the research function. The original model was successfully used in 25 Chilean universities. In this model, other inputs such as teachers, estimated in terms of their salaries, have a high correlation with the budget, thus advising against its use, as it contributes very little to the results. The model was also output-oriented, as is customary in the university sector, due to the fact that, in most cases, inputs are not controlled by the universities studied - this is more evident in public institutions whose main objective is to achieve the best possible value in outputs as they are financed by the State (Buitrago et al., 2017).

Upon justifying the orientation of the model to be used, the production frontiers of the CRS and VRS models were determined, as has been done in previous studies (García \& Palomares, 2008; Agasisti et al., 2011; Buitrago et al., 2017). Moreover, the hypothesis according to which Peruvian public universities exhibit production functions of constant returns to scale was accepted. A statistical demonstration of which of the production frontiers was the most appropriate was carried out in this research, just as in Martín (2008); other studies on efficiency 
have calculated the two types of frontier, failing to demonstrate statistically which one prevails (Haider et al., 2019). This demonstration was based on a nonparametric statistical test which allowed us to prove that CRS prevails, a model that has been employed by other authors (Pino et al., 2010).

Based on the CRS (OTE ratio) and the product-oriented model, a $56.8 \%$ of TE was estimated for public universities in Peru during 2016. Although in different contexts, this is a low value compared to that reported by other authors. For instance, Ramírez and Alfaro (2013) reported an average efficiency of $80.89 \%$ in public and private universities in Chile. Very similar values were obtained by Navarro et al. (2016) when they applied a DEA model to a group of 32 public universities in Mexico and obtained an OTE of $80.7 \%$.

As for Peru, Nunez and Cornejo (2018) conducted an efficiency study in 42 public and private universities and reported efficiency values of $55.2 \%$ and $58.9 \%$ for public universities in two out of the four DEA models they evaluated. Such values are higher than those reported for private institutions and are very similar to those obtained in our research, thereby confirming a highly heterogeneous educational sector.

The ET results also enabled the elaboration of a matrix that served as a basis for classifying institutions into four categories according to their strategic orientation (high or low) towards research or universal education. This dichotomy is very present in higher education, especially in Latin America (Cabrera et al., 2014; García de Fanelli, 2017), and, as such, has also been reported in Peru (Lavalle \& de Nicolas, 2017; Nunez \& Cornejo, 2018).

The relationship between TE of Peruvian public universities and the experience of the educational institution (seniority) was also analyzed; the result obtained by applying Spearman's Rho correlation test indicates that there is no correlation between TE of Peruvian public universities and experience, a fact that confirms the findings of Coria (2019), who found no relationship between TE of Argentine public universities and the seniority of the same.

Similarly, the relationship between TE of Peruvian public universities and the quality of the institution, based on the university ranking prepared by the CSIC (2019), was also analyzed. Spearman's Rho coefficient test was found that the TE of Peruvian public universities is not correlated to the education quality of the institution. This result disagrees with those of other researchers who reported a positive relationship between efficiency and university rankings, among them the Shanghai ranking (Blanco et al., 2019), possibly due to the fact that they evaluated the 50 best universities in the world. Likewise, Huamani et al. (2016) demonstrate the feasibility of this relationship, as the place occupied in the rankings is a solid indicator of education quality (Lavalle $\&$ de Nicolas, 2017).

Finally, an improvement plan was proposed based on the DEA methodology and can be applied as a benchmarking tool (Avkiran, 1999; Zhu, 2009, p. 131), particularly in the case of universities (Shamohammadi \& Oh, 2019). This plan was sufficiently demonstrated, as efficient universities that would function as leaders (benchmarks) for inefficient universities could be identified.

\section{CONCLUSIONS}

- Peruvian public universities have constant returns to scale production functions (average OTE $=56.8 \%$ ), used as a basis for classifying institutions into four categories according to their strategic orientations (high or low) towards research or universal education.

- Technical efficiency of Peruvian public universities is not related to the institution's experience.

- Technical efficiency of Peruvian public universities is not related to the quality of education.

- Restricted access to publications or patents developed by universities is considered a limitation and should be addressed in future research. The data are only available in the institutional portals. More inputs and outputs can also be included, in order to compare the results with those of this model involving few variables and, additionally, it is important to compare public and private education.

\section{REFERENCES}

[1] Abdurakhmanova, G., Shayusupova, N., Irmatova, A., \& Rustamov, D. (2020). The Role of the Digital Economy in the Development of the Human Capital Market. International Journal of Psychological Rehabilitation, 24(07), 8043-8051.

[2] Agasisti, T., Dal Bianco, A., Landoni, P., Sala, A., \& Salerno, M. (2011). Evaluating the Efficiency of Research in Academic Departments: An Empirical Analysis in an Italian Region. Higher Education Quarterly, 65(3), 267-289. 
Retrieved from https://doi.org/10.1111/j.14682273.2011.00489.x

[3] Arieu, A. (2004). Eficiencia técnica comparada en elevadores de granos de Argentina, bajo una aplicación de análisis de envolvente de datos. La situación del puerto de Bahía Blanca. Buenos Aires: Asociación Argentina de Economía Política. Retrieved from https:// aaep.org.ar/anales/works/works2004/Arieu.pdf

[4] Arias, P. (2019). La investigación: requisito para el avance de la calidad en las universidades peruanas. In Crescendo, 10(3), 447.

[5] Avkiran, N. (1999). An Application Reference for Data Envelopment Analysis in Branch Banking: Helping the Novice Researcher. International Journal of Bank Marketing, 17(5), 206-220. Retrieved from https://doi. org/10.1108/02652329910292675

[6] Ayaviri, V., \& Zamora, G. (2016). Medición de la eficiencia en las universidades. Una propuesta metodológica. Perspectivas, (37), 7-22.

[7] Banker, R., Charnes, A., \& Cooper, W. (1984). Some models for estimating technical and scale inefficiencies in data envelopment analysis. Management Science, 30(9), 10781092. Retrieved from https://doi.org/10.1287/ mnsc.30.9.1078

[8] Blanco, M., Bares, L., \& Hrynevych, O. (2019). Análisis de la eficiencia global de las 50 mejores universidades del mundo. Revista Espacios, 40(9), 30. Retrieved from https://www. revistaespacios.com/a19v40n09/19400930. $\mathrm{html}$

[9] Blecich, A. (2020). Factors Affecting Relative Efficiency of Higher Education Institutions of Economic Orientation. Management: Journal of Contemporary Management Issues, 25(1), 45-67. Retrieved from https://doi.org/10.30924/ mjcmi.25.1.3

[10] Buckle, R., \& Creedy, J. (2019). The Evolution of Research Quality in New Zealand Universities as Measured by the PerformanceBased Research Fund Process, New Zealand Economic Papers, 53(2), 144-165. Retrieved from https://doi.org/10.1080/00779954.2018.1 429486

[11] Buitrago O., Espitia A., \& Molano, L. (2017). Análisis envolvente de datos para la medición de la eficiencia en instituciones de educación superior: una revisión del estado del arte. Revista Científica General José María Córdova, 15(19), 147-173.
[12] Cabrera, A., Pérez, P., \& López, L. (2014). Evolución de perspectivas en el estudio de la retención universitaria en los EE. UU.: Bases conceptuales y puntos de inflexión. En P. Figuera (Ed.), Persistir con éxito en la universidad: de la investigación a la acción (pp. 15-40). Barcelona, Spain: Laertes.

[13] Campoverde, J., Romero, C., \& Borenstein, D. (2019). Evaluación de eficiencia de cooperativas de ahorro y crédito en Ecuador: aplicación del modelo Análisis Envolvente de Datos DEA. Contaduría y administración, 64(1), 1-19.

[14] Castañeda, P. (2019). Modelo de medición de la productividad para fábricas de software. (Doctoral thesis). Universidad Nacional Mayor de San Marcos, Lima.

[15] Charnes, A., Cooper, W., Lewin, A., \& Seiford, L. (1994). Data Envelopment Analysis: Theory, Methodology, and Application. Kluwer Academic Publishers, Norwell.

[16] Charnes, A., Cooper, W., \& Rhodes, E. (1978). Measuring the efficiency of decision making units. European journal of operational research, 2(6), 429-444.

[17] Coelli, T., Prasada, D., \& Battese, G. (1998). An Introduction to Productivity and Efficiency Analysis. Boston, United States: Kluwer academic publishers.

[18] Coria, M. (2019). Eficiencia técnica de las universidades argentinas de gestión estatal. Ensayos de Política Económica, 1(5), 44-64.

[19] Consejo Superior de Investigaciones Científicas (2019). Ranking Web de Universidades. Retrieved from https://www. webometrics.info/es

[20] Cooper, W., Seiford, L., \& Tone, K. (2006). Introduction to Data Envelopment Analysis and its Uses: With DEA-Solver Software and References. Springer Science \& Business Media.

[21] Díaz, J. (2008). Educación superior en el Perú: tendencias de la demanda y la oferta. En M. Benavides (Ed.), Análisis de programas, procesos y resultados educativos en el Perú: contribuciones empíricas para el debate (pp. 83-129). Lima, Peru: GRADE.

[22] Escalona, L. (2013). Eficiencia técnica para las universidades públicas venezolanas a través del modelo de análisis de datos envolventes DEA. Revista Científica "Teorías, Enfoques y 
Aplicaciones en las Ciencias Sociales", 5(12), 45-62.

[23] Farrell, M. (1957). The measurement of Productive efficiency. Journal of the Royal Statistical Society. Series A, General, 125, 252267.

[24] García, A., \& Palomares, D., (September 2426, 2008). Evaluation of Spanish Universities: Efficiency, Technology and Productivity Change [Academic paper]. Prime-Latin America Conference, México D. F., Mexico.

[25] García de Fanelli, A. (2017). Políticas públicas ante la masificación de la educación universitaria: el reto de elevar la graduación, garantizando la inclusión y la calidad. En C. Marquis (Ed.), La agenda universitaria III. Propuestas de políticas y acciones (pp. 167201). Buenos Aires, Argentina: Universidad de Palermo.

[26] Barbosa, S. (2010). Evaluación de la eficiencia de las escuelas de la Universidad Industrial de Santander aplicando análisis envolvente de datos (DEA). (Doctoral thesis). Universidad Industrial de Santander, Bucaramanga.

[27] Haider, M., Raza, Q., Jameel, S., \& Pervaiz, K. (2019). A Comparative Study of Operational Efficiency of Pakistani and Malaysian Islamic Banks: Data Envelopment Analysis Approach. Asian Economic and Financial Review, 9(5), 559-580.

[28] Huamaní, G., Huamaní, S., Salcedo, J., \& Fernández, C. (2016). Modelo de Análisis Envolvente de Datos (DEA) para evaluar la eficiencia de las escuelas profesionales de Ingeniería Industrial en el Perú con enfoque de desarrollo sostenible. Tecnia, 26(2), 2-72.

[29] Jiang, J., Lee, S., \& Rah, M. (2020). Assessing the Research Efficiency of Chinese Higher Education Institutions by Data Envelopment Analysis. Asia Pacific Education Review, 21(3), 423-440.

[30] Kumar, A., \& Thakur, R. (2019). Objectivity in Performance Ranking of Higher Education Institutions Using Dynamic Data Envelopment Analysis. International Journal of Productivity and Performance Management, 68(4), 774796.

[31] Lavalle, C., \& de Nicolas, V. L. (2017). Peru and its New Challenge in Higher Education: Towards a Research University. PloS one, 12(8). Retrieved from https://doi.org/10.1371/ journal.pone. 0182631
[32] Macilwain, C. (2010). Science Economics: What Science is Really Worth. Nature, 465(7299), 682-684. Retrieved from https://doi. org/10.1038/465682a

[33] Martín, R. (2006). La evaluación de la eficiencia técnica. Una aplicación del DEA a la Universidad de la Laguna [Scientific paper]. XV Jornadas de la Asociación de la Economía de la Educación. Granada, Spain.

[34] Martín, R. (2008). La medición de la eficiencia universitaria: una aplicación del análisis envolvente de datos. Formación Universitaria, 1(2), 17-26. Retrieved from http://dx.doi. org/10.4067/S0718-50062008000200004

[35] Martin, J., \& Roman, C. (2010). Evaluating the Service Quality of Major Air Carriers: A DEA Approach. International Journal of Applied Management Science, 2(4), 351-371.

[36] Mojahedian, M., Mohammadi, A., Abdollahi, M., Kebriaeezadeh, A., Sharifzadeh, M., Asadzandi, S., \& Nikfar, S. (2020). A Review on Inputs and Outputs in Determining the Efficiency of Universities of Medical Sciences by Data Envelopment Analysis Method. Medical Journal of the Islamic Republic of Iran (MJIRI), 34(1), 34-42. Retrieved from http://mjiri.iums. ac.ir/article-1-6288-en.html

[37] Navarro, J., Gómez, R., \& Torres, Z. (2016). Las universidades en México: una medida de su eficiencia a través del análisis de la envolvente de datos con bootstrap. Acta universitaria, 26(6), 60-69. Retrieved from https://www. redalyc.org/jatsRepo/416/41649084008/html/ index.html

[38] Nieto, L. (2016). Eficiencia y financiación en las universidades públicas españolas. (Doctoral thesis). Universidad Católica San Antonio de Murcia, Murcia.

[39] Nunez, N., \& Cornejo, G. (2018). Haciendo mucho con poco: eficiencia de la investigación científica en el Perú. Revista Espacios, 39 (26), 1-7. Retrieved from http://www.revistaespacios. com/a18v39n26/a18v39n26p07.pdf

[40] Pino, J., Solís, M., Delgado, M., \& Barea, R., (2010). Evaluación de la eficiencia de grupos de investigación mediante análisis envolvente de datos (DEA). El profesional de la información, 19(2), 160-167. Retrieved from https://doi. org/10.3145/epi.2010.mar.06

[41] Ramírez, P., \& Alfaro, J. (2013). Evaluación de la eficiencia de las universidades pertenecientes al consejo de rectores de las universidades 
chilenas: Resultados de un análisis envolvente de datos. Formación universitaria, 6(3), 31-38. Retrieved from Retrieved from http://dx.doi. org/10.4067/S0718-50062013000300005

[42] Rhodes, E. (1978). Data envelopment analysis and related approaches for measuring the efficiency of decision-making unit with application to Program follow through U.S. education. (Doctoral thesis). Carnegie-Mellon University School of Urban and Public Affair, Pittsburgh.

[43] Rojas, M. (2010). Clasificación de los grupos de investigación de la facultad de Ingeniería de la Universidad Nacional de Colombia, mediante la estimación de la eficiencia técnica utilizando análisis envolvente de datos. (Master thesis). Universidad Nacional de Colombia, Bogotá. Retrieved from https://repositorio.unal.edu.co/ bitstream/handle/unal/7466/02-822021.2010. pdf? sequence $=1$ \&isAllowed $=y$

[44] Salas-Velasco, M. (2020). The Technical Efficiency Performance of The Higher Education Systems Based on Data Envelopment Analysis with an Illustration for the Spanish Case. Educational Research for Policy and Practice, 19(2), 159-180. Retrieved from https://doi. org/10.1007/s10671-019-09254-5

[45] Schwab, K. (Ed.)(2019). The Global Competitive Report 2019. World Economic Forum. Retrieved from http://www3.weforum.org/docs/ WEF_TheGlobalCompetitivenessReport2019. pdf

[46] Shamohammadi, M. y Oh, D.-H. (2019). Measuring the Efficiency Changes of Private Universities of Korea: A Two-Stage Network Data Envelopment Analysis. Technological Forecasting and Social Change, 148, 119730. Retrieved from https://doi.org/10.1016/j. techfore.2019.119730

[47] Sigler, L. (2004). Aplicación del Data Envelopment Analysis a la producción de investigación económica en la Ciudad de México: La eficiencia relativa del CIDE, COLMEX, IPN, UAM y UNAM (1990-2002) [Lecture]. 4th International Symposium of Data Envelopment Analysis and Performance Management, Birmingham, England.
[48] SUNEDU (2018). Información estadística de universidades. Retrieved from https:// www.sunedu.gob.pe/informacion-estadisticauniversidades-licenciadas/

[49] Torres, M., Vásquez, C., Luna, M, Bucci, N., Viloria, A., \& Cabrera, D. (2019). Clustering of Top 50 Latin American Universities in SIR, QS, ARWU, and Webometrics Rankings. Procedia Computer Science, 160, 467-472. Retrieved from https://doi.org/10.1016/j. procs.2019.11.063

[50] Torres, M., Vásquez, C., Viloria, A., Borrero, T., Varela, N., Cabrera, D., Gaitán, M., \& Gutiérrez, J. (2018). Efficiency analysis of the visibility of Latin American Universities and their impact on the ranking web. In Y. Tan, Y. Shi, \& Q. Tang (Eds.), Data Mining and Big Data International Conference on Data Mining and Big Data (pp. 235-243). Cham, Switzerland: Springer. Retrieved from https://doi.org/10.1007/978-3319-93803-5_22

[51] UNESCO, UNICEF, Banco Mundial, UNFPA, PNUD, O. M. y A. (2015). Declaración de Incheon y Marco de Acción para la realización del Objetivo del Desarrollo Sostenible 4. Unescodoc, 1-84. Retrieved from https://unesdoc.unesco.org/ark:/48223/ pf0000245656_spa

[52] Wodon, Q. (2019). Measuring the Contribution of Faith-based Schools to Human Capital Wealth: Estimates for the Catholic Church. The Review of Faith \& International Affairs, 17(4), 94-102. Retrieved from https://doi.org/10.1080/ 15570274.2019.1681782

[53] Zhao, Y. (2020). Transformation of Educational Management Mode in Ocean Colleges and Universities. Journal of Coastal Research, 110(1), 67-70. Retrieved from https://doi. org/10.2112/JCR-SI110-017.1

[54] Zhu, J. (2009). Quantitative Models for Performance Evaluation and Benchmarking. Data Envelopment Analysis with Spreadsheets ( $3^{\mathrm{a}}$ ed.). USA: Springer. 


\section{APPENDIX}

Appendix 1. Characterization of universities according to MOEIN results.

\begin{tabular}{|lcccc|}
\hline \multicolumn{5}{|c|}{ Universities located in the second quadrant } \\
\hline Acronym & $\begin{array}{c}\text { Budget } \\
\text { (MM S/) }\end{array}$ & $\begin{array}{c}\text { Renacyt } \\
\text { (Nro.) }\end{array}$ & $\begin{array}{c}\text { Enrollment } \\
\text { (Nro.) }\end{array}$ & OTE \\
\hline UNAP & 77.0 & 17 & 9819 & 57.6 \\
UNT & 156.3 & 56 & 19215 & 71.8 \\
UNF-S & 9.0 & 2 & 1062 & 55.6 \\
UNS & 33.8 & 7 & 3971 & 53.5 \\
UNTRM & 43.7 & 16 & 4501 & 67.5 \\
UNMSM & 418.7 & 280 & 41011 & 100.0 \\
UNAS & 52.6 & 10 & 4033 & 41.0 \\
UNTumbes & 44.9 & 9 & 3372 & 41.8 \\
UNALM & 130.6 & 81 & 7303 & 92.8 \\
UNI & 252.8 & 70 & 12914 & 44.1 \\
UNAAA & 5.4 & 1 & 157 & 28.3 \\
\hline Average & 111.3 & 50 & 9760 & \\
\hline Total & 1224.8 & 549 & 107358 & \\
\hline \% of total & $35.9 \%$ & $71.1 \%$ & $25.1 \%$ & \\
\hline
\end{tabular}

\begin{tabular}{|lcccc|}
\hline \multicolumn{5}{|c|}{ Universities located in the first quadrant } \\
\hline Acronym & $\begin{array}{c}\text { Budget } \\
\text { (MM S/) }\end{array}$ & $\begin{array}{c}\text { Renacyt } \\
\text { (Nro.) }\end{array}$ & $\begin{array}{c}\text { Enrollment } \\
\text { (Nro.) }\end{array}$ & OTE \\
\hline UNTELS & 12.3 & 4 & 3034 & 100,0 \\
UNAMBA & 21.5 & 9 & 3298 & 86.4 \\
UNSA & 189.2 & 58 & 28520 & 73.2 \\
UNA & 177.3 & 42 & 23175 & 60.2 \\
\hline Average & 100.1 & 28 & 14507 & \\
\hline Total & 400.3 & 113 & 58027 & \\
\hline \% of total & $11.7 \%$ & $14.6 \%$ & $13.6 \%$ & \\
\hline
\end{tabular}

\begin{tabular}{|lcccc|}
\hline \multicolumn{5}{c}{ Universities located in the third quadrant } \\
\hline Acronym & $\begin{array}{c}\text { Budget } \\
\text { (MM S/) }\end{array}$ & $\begin{array}{c}\text { Renacyt } \\
\text { (Nro.) }\end{array}$ & $\begin{array}{c}\text { Enrollment } \\
\text { (Nro.) }\end{array}$ & OTE \\
\hline UNIA & 21.3 & 3 & 1299 & 31.5 \\
UNSAAC & 174.3 & 24 & 21988 & 49.7 \\
UNDC & 9.0 & 1 & 1077 & 46.4 \\
UNJBG & 74.5 & 8 & 7401 & 39.1 \\
UNC & 77.4 & 8 & 9381 & 46.3 \\
UNAM & 29.4 & 3 & 1518 & 24.8 \\
UNH & 59.4 & 6 & 6818 & 44.0 \\
UNAJMA & 15.3 & 1 & 1356 & 33.4 \\
UNSM & 61.6 & 4 & 6138 & 37.0 \\
UNACH & 16.2 & 1 & 1319 & 30.7 \\
UNU & 49.8 & 3 & 6462 & 47.0 \\
UNAJ & 20.5 & 1 & 1263 & 23.4 \\
UNICA & 118.3 & 4 & 14045 & 42.8 \\
UNE & 85.1 & 0 & 7753 & 32.9 \\
\hline Average & 58.0 & 5 & 6273 & \\
\hline Total & 812.1 & 67 & 87818 & \\
\hline$\%$ of total & $23.8 \%$ & $8.7 \%$ & $20.6 \%$ & \\
\hline
\end{tabular}

\begin{tabular}{|lcccc|}
\hline \multicolumn{5}{c|}{ Universities located in the fourth quadrant } \\
\hline Acronym & $\begin{array}{c}\text { Budget } \\
\text { (MM S/) }\end{array}$ & $\begin{array}{c}\text { Renacyt } \\
\text { (Nro.) }\end{array}$ & $\begin{array}{c}\text { Enrollment } \\
\text { (Nro.) }\end{array}$ & OTE \\
\hline UNCP & 99.9 & 13 & 24977 & 91.3 \\
UNASAM & 49.1 & 6 & 13603 & 100.0 \\
UNSCH & 74.9 & 6 & 12649 & 61.3 \\
UNPRG & 109.6 & 6 & 16618 & 54.7 \\
UNAC & 79.9 & 4 & 16921 & 76.5 \\
UNP & 145.8 & 6 & 19933 & 49.3 \\
UNDAC & 66.9 & 1 & 8825 & 47.6 \\
UNFV & 154.5 & 1 & 25201 & 58.9 \\
UNJ & 8.1 & 0 & 1783 & 79.5 \\
UNAB & 8.9 & 0 & 1835 & 74.2 \\
UNFJFSC & 80.4 & 0 & 16390 & 73.6 \\
UNHEVAL & 68.2 & 0 & 11838 & 62.7 \\
UNAMAD & 24.3 & 0 & 3475 & 51.7 \\
\hline Average & 74.7 & 3 & 13388 & \\
\hline Total & 970.5 & 43 & 174048 \\
\hline \% of total & $28.5 \%$ & $5.6 \%$ & $40.7 \%$ & \\
\hline
\end{tabular}

Total number of universities (inputs and outputs).

\begin{tabular}{|lccc|}
\hline & $\begin{array}{l}\text { Budget } \\
\text { (MM S/) }\end{array}$ & $\begin{array}{l}\text { Renacyt } \\
\text { (Nro.) }\end{array}$ & $\begin{array}{l}\text { Enrollment } \\
\text { (Nro.) }\end{array}$ \\
\hline Total & 3407.7 & 772 & 427251 \\
\hline$\%$ & $100.0 \%$ & $100.0 \%$ & $100.0 \%$ \\
\hline
\end{tabular}

Total number of universities (inputs and outputs). 
